\title{
Scalar multiplet dark matter in a fast expanding Universe: Resurrection of the desert region
}

\author{
Basabendu Barman®, ${ }^{1, *}$ Purusottam Ghosh $\odot,{ }^{2, \dagger}$ Farinaldo S. Queiroz, ${ }^{3, \sharp}$ and Abhijit Kumar Saha $\odot^{4, \S}$ \\ ${ }^{1}$ Department of Physics, IIT Guwahati, Guwahati-781039, India \\ ${ }^{2}$ Regional Centre for Accelerator-based Particle Physics, Harish-Chandra Research Institute, \\ HBNI, Chhatnag Road, Jhunsi, Allahabad-211019, India \\ ${ }^{3}$ International Institute of Physics, Universidade Federal do Rio Grande do Norte, \\ Campus Universitário, Lagoa Nova, Natal, RN 59078-970, Brazil \\ Departamento de Física, Universidade Federal do Rio Grande do Norte, 59078-970 Natal, RN, Brazil \\ ${ }^{4}$ School of Physical Sciences, Indian Association for the Cultivation of Science, \\ $2 A$ \& $2 B$ Raja S.C. Mullick Road, Kolkata 700 032, India
}

(Received 2 March 2021; accepted 8 July 2021; published 30 July 2021)

\begin{abstract}
We examine the impact of a faster expanding Universe on the phenomenology of scalar dark matter $(\mathrm{DM})$ associated with $S U(2)_{L}$ multiplets. Earlier works with a radiation dominated Universe have reported the presence of desert region for both inert $S U(2)_{L}$ doublet and triplet DM candidates where the DM is underabundant. We find that the existence of a faster expanding component before big bang nucleosynthesis can revive a substantial part of the desert parameter space consistent with relic density requirements and other direct and indirect search bounds. We also review the possible collider search prospects of the newly obtained parameter space and predict that such region might be probed at the future colliders with improved sensitivity via a disappearing/stable charged track.
\end{abstract}

DOI: $10.1103 /$ PhysRevD.104.015040

\section{INTRODUCTION}

Production of dark matter (DM) in scenarios with a nonstandard history has gained growing interest in recent times [1-36]. Since the cosmological history of the early Universe prior to big bang nucleosynthesis (BBN) is vastly dark, the possibility of presence of a nonstandard era in the early Universe is open. In fact, there are no fundamental reasons to assume that the Universe was radiation dominated (RD) in the pre-BBN regime at $t \sim 1 \mathrm{sec}$. The history of the Universe can be modeled, in general, by the presence of a fluid with arbitrary equation of state parameter, which is zero for matter domination. If the equation of state parameter of a fluid turns out to be larger than the value for radiation, then the fluid acts as a fast expanding component.

Study of DM phenomenology in the presence of a modified cosmological epoch has been performed widely, and it shows several significant observational consequences

\footnotetext{
*bb1988@iitg.ac.in

†purusottamghosh@hri.res.in

farinaldo.queiroz@iip.ufrn.br

§psaks2484@iacs.res.in
}

Published by the American Physical Society under the terms of the Creative Commons Attribution 4.0 International license. Further distribution of this work must maintain attribution to the author(s) and the published article's title, journal citation, and DOI. Funded by SCOAP.
[25,26,37]. In Ref. [27], a model-independent analysis of DM phenomenology in a fast expanding Universe is worked out. It is observed that if DM freezes out during the fast expansion period of the Universe, a larger interaction strength is required compared to that in the standard scenario (with radiation domination) to satisfy the relic abundance bound provided by the Planck experiment. At some stage during the evolution of the Universe, at least before the $\mathrm{BBN}$, the domination of the fast expanding component has to end such that the standard RD Universe takes over. A similar phenomenological study with freezein production of DM in a fast expanding Universe is explored in Ref. [28]. With the emergence of this proposal, further efforts have been put forward to cultivate the DM phenomenology considering such a nonstandard scenario in different well-established beyond standard model frameworks. For example, phenomenology of a real gauge singlet scalar DM in nonstandard cosmologies can be found in Ref. [29]. Well-motivated anatomy on the revival of the Z-boson and Higgs mediated DM model with alternative cosmology (late matter decay) is presented in Refs. [25,30]. In Refs. [13,32,33], the possibility of sterile neutrinos as dark matter candidates with modified cosmology has been discussed. Such sterile neutrinos can provide a sensitive probe of the pre-BBN epoch as pointed out in Ref. [31]. In Ref. [34], the case for fermion DM originating from a different order of multiplets is studied. 
Motivated by these, in the present work, we aim to resurrect the so-called desert region in the parameter space of the $S U(2)_{L}$ inert doublet (IDM) and triplet dark matter (ITDM) models $[38,39]$ by considering the presence of a faster expanding component (kinaton or faster than kinaton) in the early Universe. In the context of singlecomponent ${ }^{1}$ IDM dark matter, it is well known [43,44] that the intermediate DM mass regime $80 \lesssim m_{\mathrm{DM}} \lesssim$ $525 \mathrm{GeV}$ suffers from an underabundance issue. It occurs due to large interaction rate of the DM [mediated by $S U(2)_{L}$ gauge bosons] with the standard model (SM) particles, resulting in late freeze-out and subsequently less abundance. This particular mass window for IDM is thus referred as the desert in the relic density allowed parameter space for the DM. On the other hand, for single-component DM that stems from an inert scalar triplet, right relic density is achieved at a very large DM mass greater than or approximately equal to $2 \mathrm{TeV}$ under standard freeze-out assumptions. This happens due to small radiative mass splitting between the charged and neutral component of the scalar triplet (which is $\sim 166 \mathrm{MeV}$, that leads to huge coannihilation resulting in DM underabundance. Several prescriptions have been put forward for the revival of the IDM desert. These ideas basically revolve around extending the SM particle content $[44,45]$. The case for the scotogenic DM model in a modified cosmological scenario has been discussed earlier in Ref. [35]. Although authors of Ref. [35] briefly remarked on the impact of nonstandard Universe in DM relic abundance, their work is more focused on addressing neutrino mass and leptogenesis. Thus, a detailed investigation of DM phenomenology and the impact of direct, indirect, and collider searches on the DM parameter space is highly recommended.

In the first part of the work, our attempt is to make an exact prediction on the allowed parameter space of the usual IDM scenario in the presence of a fast expanding Universe. We also elucidate in detail the effect of fast expansion on the subsequent collider signature of the model. We first obtain the parameter space for the IDM dark matter that satisfies the relic abundance criteria by varying the relevant parameters that control expansion of the Universe. We find a significant part of the relic allowed parameter space further gets disfavored upon imposing the direct and indirect search constraints together with the requirement of DM thermalization, which, in turn, directly restricts the amount of fast expansion. Since the mass difference of the DM with other neutral and charged eigenstates is found to be small, the collider search of the allowed parameter space is limited and can be probed with the identification of the charged track signal of a

\footnotetext{
${ }^{1}$ In the multicomponent DM framework, individual DM candidates can be underabundant, and the desert region is thus not an issue there. Such frameworks involving multicomponent DM are proposed in Refs. [40-42].
}

long-lived charged scalar. We anticipate that the improved sensitivity of CMS/ATLAS search [46-48] can be used as an useful tool to test the early Universe history before BBN. In the later part, we extend our analysis for a $S U(2)_{L}$ triplet DM model with zero hypercharge. Similar to the IDM case, the existence of a desert region for triplet DM is mentioned in earlier works [49-57]. We use the same methodology of faster-than-usual expansion to revive part of the desert confronting all possible experimental bounds (including direct and indirect searches), which has not been done earlier to the best of our knowledge.

The paper is organized as follows. In Sec. II, we briefly sketch the nonstandard cosmological framework that arises due to fast expansion. The phenomenology for inert doublet DM in the light of fast expansion is elaborated in Sec. III, where we have discussed the modification in the Boltzmann equation due to modified Hubble rate in Sec. III A 1; Sec. III A 2 illustrates how the DM yield gets modified once fast expansion is invoked; a detailed parameter space scan showing the constraints from DM relic abundance, direct, and indirect searches is discussed in Sec. III A 3; a possible collider signature for the revived parameter space is discussed in Sec. III A 4; and the fate of scalar triplet DM in a fast expanding Universe is illustrated in Sec. III B. Finally, in Sec. IV, we conclude by summarizing our findings.

\section{NONSTANDARD SCENARIOS OF THE UNIVERSE}

Here, we briefly present the recipe to analyze the early Universe by considering both standard and nonstandard scenarios. The expansion rate of the Universe is measured by the Hubble parameter $\mathcal{H}$, which is connected to the total energy density of the Universe through the standard Friedmann equation. In the standard case, it is assumed that the Universe was radiation dominated starting from the reheating era up to BBN. Here, we assume somewhat a different possibility that the Universe before BBN was occupied by different species, namely, radiation and $\eta$, with energy densities $\rho_{\text {rad }}$ and $\rho_{\eta}$, respectively. The equation of state for a particular component is given by

$$
p=\omega \rho,
$$

where $p$ stands for the pressure of that component. For radiation, $\omega_{R}=\frac{1}{3}$, while for $\eta, \omega_{\eta}$ could be different. The $\omega_{\eta}=0$ case is familiar as early matter domination, and $\omega_{\eta}=1$ is dubbed as a fast expanding Universe. However, irrespective of the nature of $\eta$, the energy component $\rho_{\eta}$ must be subdominant compared to $\rho_{R}$ before BBN takes place. This poses a strong lower bound on the temperature of the Universe $T \gtrsim(15.4)^{1 / n} \mathrm{MeV}$ before the onset of BBN (see Appendix B). Considering the presence of a new species $(\eta)$ along with the radiation field, the total energy 
budget of the Universe is $\rho=\rho_{\text {rad }}+\rho_{\eta}$. For standard cosmology, the $\eta$ field would be absent, and we simply write $\rho=\rho_{\text {rad }}$. One can always express the energy density of the radiation component, which is given by a function of temperature,

$$
\rho_{\mathrm{rad}}(T)=\frac{\pi^{2}}{30} g_{*}(T) T^{4}
$$

where $g_{*}(T)$ stands for the effective number of relativistic degrees of freedom at temperature $T$. In the limit of entropy conservation per comoving volume, i.e., $s a^{3}=$ const, one can define $\rho_{\text {rad }}(t) \propto a(t)^{-4}$. Now, in the case of a faster expansion of the Universe, the energy density of $\eta$ field is anticipated to be redshifted more rapidly than the radiation. Accordingly, one can obtain $\rho_{\eta} \propto a(t)^{-(4+n)}$ with $n>0$.

The entropy density of the Universe is parametrized as $s(T)=\frac{2 \pi^{2}}{45} g_{* s}(T) T^{3}$, where $g_{* s}$ is the effective relativistic degrees of freedom that contribute to the entropy density. Utilizing the energy conservation principle, a general form of $\rho_{\eta}$ can be constructed as

$$
\rho_{\eta}(T)=\rho_{\eta}\left(T_{R}\right)\left(\frac{g_{* s}(T)}{g_{* s}\left(T_{R}\right)}\right)^{(4+n) / 3}\left(\frac{T}{T_{R}}\right)^{(4+n)} .
$$

The temperature $T_{R}$ is an unknown parameter (greater than $T_{\mathrm{BBN}}$ ) and can be safely assumed as the point of equality of two respective energy densities: $\rho_{\eta}\left(T_{R}\right)=\rho_{\text {rad }}\left(T_{R}\right)$. Using this criteria, it is simple to specify the total energy density at any temperature $\left(T>T_{R}\right)$ as [27]

$$
\begin{gathered}
\rho(T)=\rho_{\mathrm{rad}}(T)+\rho_{\eta}(T) \\
=\rho_{\mathrm{rad}}(T)\left[1+\frac{g_{*}\left(T_{R}\right)}{g_{*}(T)}\left(\frac{g_{* s}(T)}{g_{* s}\left(T_{R}\right)}\right)^{(4+n) / 3}\left(\frac{T}{T_{R}}\right)^{n}\right] .
\end{gathered}
$$

From the above equation, it is evident that the energy density of the Universe at any arbitrary temperature $\left(T>T_{R}\right)$ is dominated by the $\eta$ component. Now, the standard Friedmann equation connecting the Hubble parameter with the energy density of the Universe is given by

$$
\mathcal{H}^{2}=\frac{\rho}{3 M_{\mathrm{Pl}}^{2}},
$$

with $M_{\mathrm{Pl}}=2.4 \times 10^{18} \mathrm{GeV}$ being the reduced Planck mass. At temperature higher than $T_{R}$ with the condition $g_{*}(T)=\bar{g}_{*}$, which can be considered to be some constant, the Hubble rate can approximately be recast into the form [27]

$$
\begin{aligned}
\mathcal{H}(T) & \left.\approx \frac{\pi \bar{g}_{*}^{1 / 2}}{3 \sqrt{10}} \frac{T^{2}}{M_{\mathrm{Pl}}}\left(\frac{T}{T_{R}}\right)^{n / 2}, \quad \text { (with } \quad T \gg T_{R}\right) \\
& =\mathcal{H}_{R}(T)\left(\frac{T}{T_{R}}\right)^{n / 2}
\end{aligned}
$$

where $\mathcal{H}_{R}(T) \simeq 0.33 \bar{g}_{*}^{1 / 2} \frac{T^{2}}{M_{\mathrm{Pl}}}$, the Hubble rate for radiation dominated Universe. In the case of the SM, $\bar{g}_{*}$ can be identified with the total SM degrees of freedom $g_{*}(\mathrm{SM})=106.75$. It is important to note from Eq (7) that the expansion rate is larger than what it is supposed to be in the standard cosmological background provided $T>T_{R}$ and $n>0$. Hence, it can be stated that if the DM freezes out during $\eta$ domination the situation will alter significantly with respect to the one in the standard cosmology. Finally, it is worth noting that $T_{R}$ cannot be too small such that it alters the standard BBN. For a certain value of $n$, BBN constraints provide a lower limit on $T_{R}$, which we report in Appendix B:

$$
T_{R} \gtrsim(15.4)^{1 / n} \mathrm{MeV} \text {. }
$$

To this end, we have assumed the prescription for DM freeze-out in a fast expanding Universe in a model-agnostic way. Before moving on to the next section, we would like to provide few examples in which it is possible to have some physical realization of the new species $\eta$. We consider $\eta$ to be a real scalar field minimally coupled to gravity. In that case, a specific form for $\omega(=p / \rho)$ can be written as

$$
\omega=\frac{\frac{1}{2}\left(\frac{d \eta}{d t}\right)^{2}-V(\eta)}{\frac{1}{2}\left(\frac{d \eta}{d t}\right)^{2}+V(\eta)} .
$$

The energy density of $\eta$ redshifts as [28]

$$
\rho_{\eta} \propto a^{-3(1+\omega)},
$$

which can be converted to $\rho_{\eta} \propto a^{-4+n}$ with $\omega=\frac{1}{3}(n+1)$. For a positive scalar potential, two possible extreme limits are $\left(\frac{d \eta}{d t}\right)^{2} \ll V(\eta)$ or the $\left(\frac{d \eta}{d t}\right)^{2} \gg V(\eta)$. These correspond to $\omega \in(-1,+1)$ leading to $n \in(-4,+2)$. The $n=2$ case is realized for a Universe dominated by kinaton, which can be identified with a quintessence fluid [58,59]. For theories with $n>2$, one has to consider scenarios faster than quintessence with negative potential. Examples of such theories can be found in Refs. [60,61], in which one assumes the presence of a pre-big bang "ekpyrotic" phase. The key ingredient of ekpyrosis is the same as that of inflation, namely, a scalar field rolling down some selfinteraction potential. However, the crucial difference is that, while inflation requires a flat and positive potential, its ekpyrotic counterpart is steep and negative. Note that, in 
this work, we consider the kination or faster than kination scenario with $n \geq 2$.

\section{SCALAR MULTIPLET DARK MATTER IN A FAST EXPANDING UNIVERSE}

In this section, we perform the phenomenological analysis of DM belonging to different representation of scalar multiplets when the Hubble parameter is modified under the assumption of faster-than-usual expansion in the pre-BBN era. Our analysis, as mentioned in the Introduction, addresses two well-motivated DM scenarios:

(i) the inert doublet model, where the second Higgs doublet carries a nonzero hypercharge and the DM emerges either as the $C P$-even or as the $C P$-odd component of the second Higgs,

(ii) a hyperchargeless $(Y=0)$ inert triplet scalar under $S U(2)_{L}$ where the neutral component of the scalar triplet can be a viable DM candidate. We shall call this the inert triplet dark matter.

In either case, one has to impose a discrete symmetry to ensure the stability of the DM. The DM phenomenology for both of these models has been studied in great detail in the background of a standard radiation-dominated Universe. From this analyses, it has been found that for the case of IDM the DM mass range $m_{W}(\sim 80) \lesssim m_{\mathrm{DM}} \lesssim 525 \mathrm{GeV}$ is underabundant, while for ITDM, the DM lighter than $1.9 \mathrm{TeV}$ is underabundant. Here, we would like to mention that another possibility of having a scalar triplet DM is to consider a $Y=2$ triplet; however, for such a nonzero hypercharge multiplet, a $Z$-mediated direct detection bound becomes severe, making most of the DM parameter space forbidden simply from the spin-independent direct detection bound $[42,50,62]$. Therefore, we shall focus only on the $Y=0$ triplet. Our goal is, as emphasized earlier, to see how much of the parameter space ruled out by the standard cosmological background can be revived under the assumption of fast expansion without extending the particle spectrum for each of these models further. In the following sections, we shall furnish the details of the models and explicitly demonstrate how the nonstandard cosmological scenario drastically alters the standard picture.

\section{A. Inert doublet model}

Here, we would like to briefly summarize the inert doublet model (IDM) framework. The IDM consists of an extra scalar that transforms as a doublet under the SM gauge symmetry. An additional $Z_{2}$ symmetry is also imposed, under which all the SM fields are even while the inert doublet transforms nontrivially. This discrete symmetry remains unbroken since it is assumed that the extra scalar does not acquire a vacuum expectation value (VEV). With this minimal particle content, the scalar potential takes the form [43,44,63-68]

$$
\begin{aligned}
V(H, \Phi)= & -\mu_{H}^{2}|H|^{2}+\lambda_{H}|H|^{4}+\mu_{\Phi}^{2}\left(\Phi^{\dagger} \Phi\right)+\lambda_{\Phi}\left(\Phi^{\dagger} \Phi\right)^{2} \\
& +\lambda_{1}\left(H^{\dagger} H\right)\left(\Phi^{\dagger} \Phi\right)+\lambda_{2}\left(H^{\dagger} \Phi\right)\left(\Phi^{\dagger} H\right) \\
& +\frac{\lambda_{3}}{2}\left[\left(H^{\dagger} \Phi\right)^{2}+\text { H.c. }\right] .
\end{aligned}
$$

$m_{h}=125.09 \mathrm{GeVAfter}$ electroweak symmetry breaking the SM-like Higgs doublet acquires nonzero vacuum expectation value. Considering the unitary gauge, the two scalar doublets can be expressed as

$$
H=\left(\begin{array}{c}
0 \\
\frac{h+v}{\sqrt{2}},
\end{array}\right), \quad \Phi=\left(\begin{array}{c}
H^{ \pm} \\
\frac{H^{0}+i A^{0}}{\sqrt{2}},
\end{array}\right),
$$

where $v=246 \mathrm{GeV}$ is the SM Higgs VEV. After minimizing the potential along different field directions, one can obtain the relations between the physical masses and the associated couplings,

$$
\begin{aligned}
\mu_{H}^{2} & =\frac{m_{h}^{2}}{2}, \quad \mu_{\Phi}^{2}=m_{H^{0}}^{2}-\lambda_{L} v^{2}, \quad \lambda_{3}=\frac{1}{v^{2}}\left(m_{H^{0}}^{2}-m_{A^{0}}^{2}\right), \\
\lambda_{2} & =\frac{1}{v^{2}}\left(m_{H^{0}}^{2}+m_{A^{0}}^{2}-2 m_{H^{ \pm}}^{2}\right), \\
\lambda_{1} & =2 \lambda_{L}-\frac{2}{v^{2}}\left(m_{H^{0}}^{2}-m_{H^{ \pm}}^{2}\right),
\end{aligned}
$$

where $\lambda_{L}=\frac{1}{2}\left(\lambda_{1}+\lambda_{2}+\lambda_{3}\right)$. The mass eigenvalues of the three neutral scalars are indicated by $m_{h}, m_{H^{0}}, m_{A^{0}}$. We dene the SM model like $C P$-even Higgs by $h$ with mass, while $H^{0}$ is the additional $C P$-even Higgs that can be heavier or lighter than the SM one. The only $C P$-odd Higgs in the set-up is identied with $A^{0}$. The $m_{H^{ \pm}}$denotes the mass of charged scalar eigenstate(s). In our case, we consider $H^{0}$ to be the DM candidate with mass $m_{H^{0}}$ which automatically implies $m_{H^{0}}<m_{A^{0}, H^{ \pm}}$. We also assume

$$
\Delta M=m_{A^{0}}-m_{H^{0}}=m_{H^{ \pm}}-m_{H^{0}}
$$

to reduce the number of free parameters. ${ }^{2}$ Now, the masses and couplings are subject to a number of theoretical and experimental constraints. Below, we briefly mention them:

Vacuum stability.-Stability of the Two Higgs doublet model potential is ensured by the following conditions [69,70]:

$$
\begin{aligned}
& \lambda_{H}, \lambda_{\Phi}>0 ; \quad \lambda_{1}+2 \sqrt{\lambda_{H} \lambda_{\Phi}}>0 ; \\
& \lambda_{1}+\lambda_{2}-\left|\lambda_{3}\right|+2 \sqrt{\lambda_{H} \lambda_{\Phi}}>0 .
\end{aligned}
$$

These conditions are to ensure that the scalar potential is bounded from below.

Perturbativity.-Tree-level unitarity imposes bounds on the size of the quartic couplings $\lambda_{i}$ or various combinations of them [63]. On top of that, the theory remains perturbative at any given scale if naively

\footnotetext{
${ }^{2}$ Choosing $m_{A^{0}} \neq m_{H^{ \pm}}$does not alter our conclusions.
} 


$$
\left|\lambda_{i}\right| \lesssim 4 \pi, \quad i=1,2,3, H, \Phi .
$$

In view of the unitarity bound, we shall keep the magnitudes of all the relevant couplings below the order of unity.

Oblique parameters. - The splitting between the heavy scalar masses is constrained by the oblique electroweak $T$ parameter [71] whose expression in the alignment limit is given by [72]

$$
\Delta T=\frac{g_{2}^{2}}{64 \pi^{2} m_{W}^{2}}\left\{\zeta\left(m_{H^{ \pm}}^{2}, m_{A}^{2}\right)+\zeta\left(m_{H^{ \pm}}^{2}, m_{H}^{2}\right)-\zeta\left(m_{A}^{2}, m_{H}^{2}\right)\right\},
$$

with

$$
\zeta(x, y)= \begin{cases}\frac{x+y}{2}-\frac{x y}{x-y} \ln \left(\frac{x}{y}\right), & \text { if } x \neq y . \\ 0, & \text { if } x=y .\end{cases}
$$

The contribution to the $S$ parameter is always small [72] and can safely be neglected. We thus concentrate on the $T$ parameter only, which is bounded by the global electroweak fit results [73] as

$$
\Delta T=0.07 \pm 0.12
$$

It can be understood from Eq. (18) that the constraints on the oblique parameter typically prohibit large mass splittings among inert states. However, we shall see that to satisfy the other DM related constraints in general, relatively small mass splittings are required and hence the model easily bypasses the bounds arising from electroweak parameters.

Collider bounds.-To remain in compliance with the $Z$ decay width measured from LEP-II [74,75], the new scalars should obey the inequality $m_{Z}<m_{H^{0}}+m_{A^{0}}$. The LEP experiments have performed direct searches for charged Higgs. A combination of LEP data from searches in the $\tau \nu$ and $c s$ final states demands $m_{H^{+}} \gtrsim 80 \mathrm{GeV}$ under the assumption that the decay $H^{ \pm} \rightarrow W^{ \pm} h$ is absent [74,75]. As discussed in Ref. [76], Run-I of the LHC provides relevant constraints on the IDM model that significantly extend previous limits from LEP. Run-1 of ATLAS dilepton searches exclude, at $95 \%$ C.L., inert scalar masses up to about $35 \mathrm{GeV}$ for pseudoscalar masses around $100 \mathrm{GeV}$, with the limits becoming stronger for larger $m_{A^{0}}$ [76]. Also, for $m_{H^{0}}<m_{h} / 2$, the SM-like $C P$ even Higgs can decay invisibly to a pair of inert DM, which is also constrained from the invisible Higgs decay width measurement at the LHC [73].

\section{IDM dark matter in light of fast expansion}

As stated earlier, we refer the intermediate DM mass range $m_{W} \lesssim m_{H^{0}} \lesssim 525 \mathrm{GeV}$ as the IDM desert where the observed relic abundance of the DM cannot be generated as the DM annihilation cross section is more than what is required to produce correct abundance through the vanilla freeze-out mechanism. The inert doublet DM can (co) annihilate to SM states through both Higgs and $Z, W^{ \pm}$mediated processes. The dominant contribution to the DM abundance generally comes from the DM pair annihilation to gauge boson final states irrespective of the choice of $\Delta M$. Although coannihilation of DM with its charged counterpart $H^{ \pm}$turns out to be important for small $\Delta M \sim 1 \mathrm{GeV}$, it provides subdominant contribution to the relic abundance as we have checked. Because of large annihilation rates (involving gauge interactions), the DM is underabundant within this mass range. Without extending the model further or resorting to other DM production mechanisms, our aim is to revive the desert region with the help of nonstandard cosmology.

The Boltzmann equation (BEQ) that governs the evolution of the comoving number density of the DM, in the standard radiation dominated Universe, has the familiar form [77]

$$
\frac{d Y_{\mathrm{DM}}}{d x}=-\frac{\langle\sigma v\rangle s}{\mathcal{H}_{R}(T) x}\left(Y_{\mathrm{DM}}^{2}-Y_{\mathrm{DM}}^{\mathrm{eq}^{2}}\right),
$$

where $x=\frac{m_{H^{0}}}{T}$ and $\langle\sigma v\rangle$ stands for the thermally averaged annihilation cross section. It is always convenient to recast the DM number density in terms of the dimensionless quantity $Y_{\mathrm{DM}}=n_{\mathrm{DM}} / s$ with $s$ being the entropy per comoving volume. The equilibrium number density of the DM component, in terms of the yield $Y$, is given by

$$
Y_{\mathrm{DM}}^{\mathrm{eq}}=\frac{45}{4 \pi^{4}}\left(\frac{g_{\mathrm{DM}}}{g_{* s}}\right) x^{2} K_{2}(x),
$$

where $K_{2}(x)$ is the reduced Bessel function of the second kind. For the fast expanding Universe, $\mathcal{H}_{R}$ in Eq. (20) will be replaced by $\mathcal{H}$ of Eq. (7), leading to

$$
\frac{d Y_{\mathrm{DM}}}{d x}=-\frac{A\langle\sigma v\rangle}{x^{2-n / 2} \sqrt{x^{n}+\left(\frac{m_{\mathrm{DM}}}{T_{R}}\right)^{n}}}\left(Y_{\mathrm{DM}}^{2}-Y_{\mathrm{DM}}^{\mathrm{eq}}\right)
$$

with $A=\frac{2 \sqrt{2} \pi}{3 \sqrt{5}} \frac{g_{* s}}{\sqrt{g_{*}}} M_{\mathrm{pl}} m_{\mathrm{DM}}$. This is the BEQ of our interest.

As clarified before, in presence of the species $\eta$ with $n>0$, the freeze-out of DM occurs at earlier times compared to the case for the radiation-dominated Universe. In post-freeze-out time, the DM number density still keeps decreasing due to faster redshift of the energy density of $\eta$ and the constant attempt of the DM to go back to thermal equilibrium till the Universe reaches radiation domination, and finally the rate of interaction $\Gamma_{\mathrm{DM}} \ll \mathcal{H}_{R}$. The rate of decrease of the DM relic abundance in this phase is rapid for larger $n$. An approximate analytical solution for the DM yield considering $s$-wave annihilation in this regime reads 
$Y_{\mathrm{DM}}(x) \simeq \begin{cases}\frac{x_{r}}{A\langle\sigma v\rangle}\left[\frac{2}{x_{f}}+\log \left(x / x_{f}\right)\right]^{-1}, & (n=2) \\ \frac{x_{r}^{n / 2}}{2 A\langle\sigma \nu\rangle}\left[x_{f}^{n / 2-2}+\frac{x^{n / 2-1}-x_{f}^{n / 2-1}}{n-2}\right]^{-1} & (n \neq 2)\end{cases}$

as reported in Appendix A with $x_{f(r)}=m_{\mathrm{DM}} / T_{f(R)}$. It is evident from Eq. (23) that for $n=2$ after freeze-out one can observe the slow logarithmic decrease (although faster than the usual scenario) in the DM yield. The slow logarithmic decrease in the number density is the result of the relentless effort of the DM to go back to the thermal equilibrium. ${ }^{3}$ This behavior continues till $T \simeq T_{R}$, after which the Universe becomes radiation dominated and the DM comoving number density attains a constant value. For $n>2$, the effect of fast expansion is even more pronounced as the DM yield has a pure power-law dependence instead of a logarithmic one. The same as before, the DM number density keeps decreasing until radiation takes over. Similar behavior can be seen for $p$-wave annihilation as elaborated in Ref. [27]. For different choices of the relevant parameters, we shall solve Eq. (22) numerically to obtain the DM relic abundance via

$$
\Omega_{\mathrm{DM}} h^{2}=2.82 \times 10^{8} m_{H^{0}} Y_{\mathrm{DM}}(x=\infty) .
$$

This brings us to the independent parameters for IDM dark matter model in a fast expanding Universe that is going to affect the DM relic abundance:

$$
\left\{m_{H^{0}}, \Delta M, \lambda_{L}, n, T_{R}\right\}
$$

Note that the presence of last two parameters is due to consideration of fast expansion.

Apart from the requirement of obtaining the Planck observed relic abundance $\left(\Omega_{\mathrm{DM}} h^{2}=0.120 \pm 0.001\right.$ at $90 \%$ C.L. [78]), there are two other sources that impose severe bounds on the IDM desert region. The spin-independent direct search puts a stringent bound on the IDM parameter space by constraining the DM-nucleon direct detection cross section. At the tree level, the DM-nucleon scattering cross section mediated by the SM-like Higgs boson reads [79]

$$
\sigma_{n-H^{0}}^{\mathrm{SI}}=\frac{\lambda_{L}^{2} f_{N}^{2}}{\pi} \frac{\mu^{2} m_{n}^{2}}{m_{h}^{4} m_{H^{0}}^{2}},
$$

where $f_{N}=0.2837$ represents the form factor of nucleon, $m_{n}=0.939 \mathrm{GeV}$ denotes the nucleon mass, and $\mu=$ $m_{n} m_{H^{0}} /\left(m_{n}+m_{H^{0}}\right)$ is the DM-nucleon reduced mass. The spin-independent direct search exclusion limit puts bounds on the model parameters, especially on the coupling $\lambda_{L}$ and DM mass $m_{H^{0}}$ via Eq. (26), which in turn restricts the relic density allowed parameter space to remain

\footnotetext{
${ }^{3}$ This feature has been referred to as "relentless" DM in Ref. [27].
}

viable within the direct search limit. In our work, we shall consider the recent XENON1T bound [80] to restrict the parameter space wherever applicable.

The second most rigorous bound arises from the indirect search experiments that look for astrophysical sources of SM particles produced through DM annihilations or via DM decays. Among these final states, the neutral and stable particles, e.g., photon and neutrinos, can reach indirect detection detectors without getting affected much by intermediate regions. If the emitted photons lie in the gamma-ray regime, that can be measured at space-based telescopes like the Fermi-LAT [81] or ground-based telescopes like MAGIC [82]. Now, it turns out that for a singlecomponent IDM candidate, the indirect search severely restricts the thermal average cross section of $H^{0} H^{0} \rightarrow$ $W^{+} W^{-}$annihilation process. Since the bounds on other annihilation processes of IDM DM candidates are comparatively milder, we shall mostly focus into the bound on $W^{+} W^{-}$final states for constraining the parameter space. Equipped with these, we now move on to investigate the fate of the IDM desert under the influence of fast expansion.

\section{IDM dark matter yield in fast expanding background}

As stated earlier, we work in the standard freeze-out regime where we solve Eq. (20) with the assumption that the DM was in thermal equilibrium in the early Universe. To illustrate the effect of modified BEQ on the DM abundance, we deliberately consider a few benchmark values such that they provide underabundance in case of standard Universe (RD), thus falling into the desert region. Before delving into the parameter scan, we would first like to demonstrate the effect of fast expansion, i.e., the parameters $\left\{n, T_{R}\right\}$ on the DM yield. To do that, we fix the coupling $\lambda_{L}=0.01$ and choose several values of $\left\{m_{H^{0}}, \Delta M, n, T_{R}\right\}$ and obtain resulting DM yield by solving Eq. (22) numerically as stated earlier. As we shall see later, such a choice of $\lambda_{L}$ keeps the DM safe from spinindependent (SI) direct search exclusion limits. We have used the publicly available code micromeGas [83] for obtaining the annihilation cross section $\langle\sigma v\rangle$ and fed them to the modified BEQ in Eq. (22) to extract the DM yield:

(i) For the benchmark values $m_{H^{0}}=300 \mathrm{GeV}$ and $\Delta M=1 \mathrm{GeV}$, we fix $T_{R}=3 \mathrm{GeV}$. In the left panel of Fig. 1, we show the evolution of DM abundance as a function of $x=m_{H^{0}} / T$. The solid red colored curve is the case of the standard RD Universe $(n=0)$ that clearly shows the DM relic is underabundant for the chosen benchmark. As we increase the value of $n$ from zero, the final relic abundance gets enhanced obeying Eq. (24), and for $n=2$, the relic abundance is satisfied. This typical behavior surfaces because of the presence of fast expanding component $\eta$ during the DM freeze-out. Since the Hubble is larger than that in the RD Universe, the 


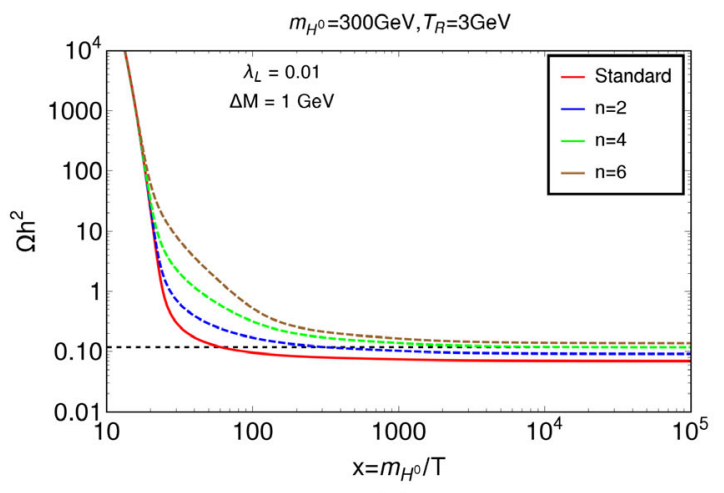

(a)

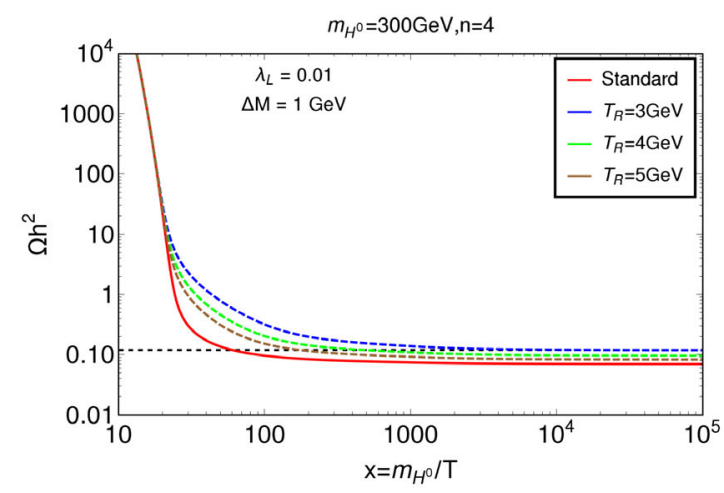

(b)

FIG. 1. (a): Evolution of DM relic abundance as function of $x=m_{H^{0}} / T$ for RD Universe (red) and in the presence of fast expansion for different values of $n(>0)$. The analysis is for a fixed $T_{R}=3 \mathrm{GeV}, \Delta \mathrm{M}=1 \mathrm{GeV}$ and $m_{H^{0}}=300 \mathrm{GeV}$ with different choices of $n=\{2,4,6\}$ shown in blue, green, and brown, respectively. (b): The DM relic density as a function of $x$ is plotted for a fixed $n=4$ for different choices of $T_{R}=\{3,4,5\} \mathrm{GeV}$ shown via the blue, green, and brown curves, respectively. In both the plots, the red solid curve corresponds to usual RD Universe with $n=0$, and the thick dashed straight line indicates the central value of the observed DM relic abundance.

DM freezes out earlier and causes an overproduction of relic that can be tamed down by suitable choice of the free parameters $\left\{n, T_{R}\right\}$. It is needless to mention that $n \rightarrow 0$ for a fixed $T_{R}$ simply reproduces the RD scenario with the unmodified Hubble rate.

(ii) Next, in the right panel of Fig. 1, we fix $n=4$ for the same DM mass of $m_{H^{0}}=300 \mathrm{GeV}$ and choose different values of $T_{R}$. As one can see from the left panel of Fig. 1, $n=4$ corresponds to DM overabundance for $T_{R}=3 \mathrm{GeV}$ (shown by the green dashed curve). To obtain the right abundance for $n=4$, one then has to go to a larger $T_{R}$ obeying Eq. (24) to tame down the Hubble rate. This is exactly what we see here. The correct DM abundance is achieved for $T_{R}=5 \mathrm{GeV}$ with $n=4$. Increasing $T_{R}$ further shall make the DM underabundance as $T_{R} \rightarrow \infty$ for a fixed $n$ correspond to the standard RD scenario.

We thus see the general trend here that when we invoke fast expansion through the Hubble parameter then for certain choices of $\left\{n, T_{R}\right\}$ it is indeed possible to revive the region of the DM parameter space that is otherwise underabundant (shown by the red curve in each plot). Our next task is to see the relic density allowed parameter space that survives once direct and indirect search bounds are imposed.

Before we proceed, it is necessary to check whether the DM everthermalizes in the fast expanding Universe at some early time validating the BEQ [Eq. (22)] that we are using to find its yield. Thermalization can be accomplished by satisfying the condition $\Gamma_{\text {int }}>\mathcal{H}(T)$ at some high temperature above the weak scale [approximately $\mathcal{O}(1) \mathrm{TeV}$ ] Considering the temperatures larger than the DM mass, the scattering rate of the DM can be approximated as [27]

$$
\Gamma_{\mathrm{int}}=n_{\mathrm{DM}}\langle\sigma v\rangle \simeq \frac{\zeta(3) T^{3}}{2 \pi^{2}} \frac{g_{2}^{4}}{32 \pi} \frac{T^{2}}{\left(T^{2}+M_{\mathrm{med}}^{2}\right)^{2}},
$$

where $g_{2}$ is the $S U(2)_{L}$ gauge coupling and $M_{\text {med }}$ is the mediator mass. ${ }^{4}$ For the inert doublet model, in principle, $\lambda_{L}$ (one of the scalar couplings) should also enter into Eq. (27) Now, since $\lambda_{L} \ll g_{2}$ (motivated from satisfying the direct search bound) always holds in our analysis, we have found that the DM pair annihilation is always dominated by the gauge boson final state, which is proportional to the coupling strength $g_{2}^{4}[43,63]$. In Fig. 2, we compare the modified Hubble rate with the DM interaction rate as a function of temperature $T$, considering $T_{R}=1 \mathrm{GeV}$ for different values of $n$. In the left panel of Fig. 2, we consider the approximate analytical relation in Eq. (27), while for the right panel, we calculate the DM interaction rate numerically by evaluating the annihilation cross section using micrOMEGAS [84] for a DM of mass $m_{H^{0}}=350 \mathrm{GeV}$. We notice the approximate expression closely follows the numerically obtained result, implying the annihilation rate of DM is largely independent of its mass. From these plots, we see, for $n=6$, thermalization is achieved at temperature $T \gtrsim 2.5 \mathrm{TeV}$ for $T_{R}=1 \mathrm{GeV}$. For $T_{R}>1 \mathrm{GeV}$, the DM thermalizes much earlier (at a larger temperature) as the modified Hubble rate decreases following Eq. (7) and it could allow higher $n>6$ values. The same conclusion can be drawn for the case of inert triplet DM where the dominant annihilation channel is again due to gauge boson final states, and hence determined by the $S U(2)_{L}$ gauge coupling. In case where the DM interaction rate is always below the Hubble rate, the thermal production of the DM is not possible, and we need to opt for the nonthermal case with modified Boltzmann equations. Taking thermalization

\footnotetext{
${ }^{4}$ For point interaction, we can consider $M_{\text {med }} \rightarrow 0$.
} 

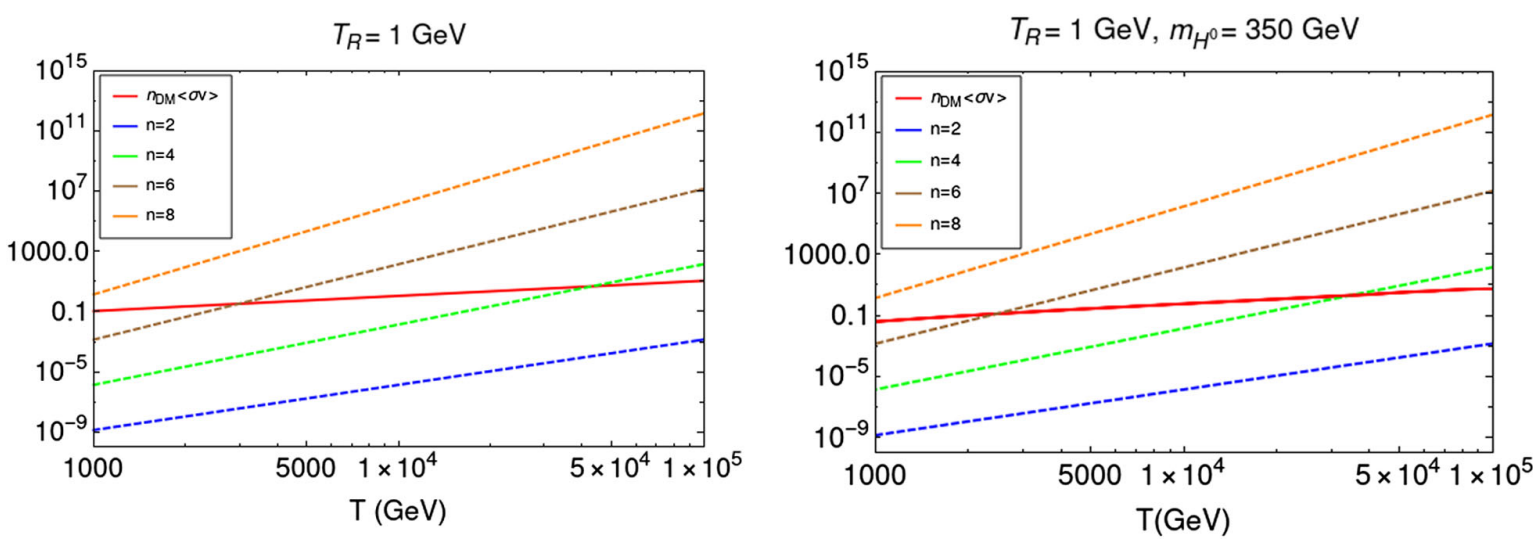

FIG. 2. The modified Hubble rates (dashed lines) are plotted as function of temperature for different values of $n$. The red solid line indicates the DM interaction rate $\Gamma_{\text {int }}$ (see the text for details) as a function of temperature $T$ of the Universe. The figure in the left panel is obtained using Eq. (27), while for right panel, we obtain the thermally averaged cross-section numerically to determine the DM interaction cross section.

of the DM in the early Universe into account, we confine ourselves within the range $2 \leq n \leq 6$ with $T_{R} \gtrsim 1 \mathrm{GeV}$, unless otherwise mentioned explicitly. ${ }^{5}$ We find, within the said range of $n, T_{R}$, that both inert doublet and inert triplet $\mathrm{DM}$ achieves thermal equilibrium for the mass ranges $m_{\mathrm{DM}} \lesssim 525 \mathrm{GeV}$ and $m_{\mathrm{DM}} \lesssim 1.9 \mathrm{TeV}$, respectively, at a temperature above the weak scale.

\section{Allowed parameter space for IDM dark matter in a fast expanding Universe}

To find out how much of the relic density allowed parameter space is left in a fast expanding framework after satisfying (in)direct detection bounds, we would like to perform a scan over the relevant parameter space. To do that, first we fix $\lambda_{L}=0.01$ as before. In that case, the remaining parameters relevant for DM phenomenology are $\left\{m_{H^{0}}, \Delta M, n, T_{R}\right\}$. In Fig. 3, we show the relic satisfied points in the $\Delta M-m_{H^{0}}$ plane by varying $n$ considering $T_{R}=\{3,4,5\} \mathrm{GeV}$. The cyan coloured region in Fig. 3 violates the indirect-detection bounds from the Fermi-LAT $W W$ nal state, and hence disfavored. For a constant $\Delta M$ and $T_{R}$, notice that a larger value of $n$ requires smaller DM mass to satisfy the relic bound. This particular nature appears since larger $n$ leads to enhanced expansion rate of the Universe, and hence the DM annihilation rate should be sufficient enough to avoid early freeze-out and subsequently overabundance. Thus, a smaller value of $m_{H^{0}}$ is preferred to be within the relic limit since the annihilation rate of the DM goes roughly as $\langle\sigma v\rangle \propto 1 / m_{H^{0}}^{2}$. However, such a requirement of the enhanced annihilation cross section due to larger $n$ may get disfavored by the indirect search bound as one can see in the leftmost panel of Fig. 3. This can be evaded if we increase the $T_{R}$ as well, since then

\footnotetext{
${ }^{5}$ Lowering $T_{R}(<1 \mathrm{GeV})$ disallows higher $n$ values from the requirement of thermalization above the weak scale.
}

it reduces the Hubble rate against larger $n$ following Eq. (7). Such a pattern can be observed in the other two figures for $T_{R}=\{4,5\} \mathrm{GeV}$. The bound arising from spin-independent direct-detection cross section for $\lambda_{L}=0.01$ is weak and does not appear in Fig. 3.

For clear insight on the detection prospect of the DM at indirect-detection experiments, in Fig. 4, we estimate the numerical values of $\langle\sigma v\rangle$ for $W^{+} W^{-}$final states of the relic satisfied points as shown earlier in Fig. 3. The latest exclusion bound from Fermi experiment due to nonobservation of the DM signal is shown via the solid black line. In accordance with the earlier trend, it can be seen that increasing $T_{R}$ reduces the $\langle\sigma v\rangle$ for a particular $n$. Hence, improved sensitivity of the Fermi experiment has the ability to probe or rule out the cases particularly with low $T_{R}$ values. So far, we have worked with $\lambda_{L}=0.01$. We would now like to see the consequence of a relatively larger $\lambda_{L}=$ 0.05 on the DM phenomenology. As is evident from Eq. (26), the direct detection cross section becomes important for a larger $\lambda_{L}$. In Fig. 5, we present the relic satisfied points in the bidimensional plane of $M_{H^{0}}-\Delta M$ for different sets of $\left\{n, T_{R}\right\}$ values. As expected, we find that for $\lambda_{L}=0.05$ the spin-independent direct detection constraints become dominant over the indirect-detection ones in the mass region $m_{H^{0}} \lesssim 480 \mathrm{GeV}$. The characteristics of the relic satised contours are the same as those portrayed for the case with $\lambda_{L}=0.01$ corresponds lower value of $T_{R}$ with other parameters are fixed. As we see, for larger $T_{R}$ and smaller $n$, the relic satisfied points with $0.01 \lesssim \Delta M \lesssim 10 \mathrm{GeV}$ are unconstrained from both direct and indirect detection. More precisely, $\Delta M<3 \mathrm{GeV}$ is ruled out for $T_{R}=3 \mathrm{GeV}$ and $n=4$, but on increasing $T_{R}$ to $8 \mathrm{GeV}$, the bound on $\Delta M$ is significantly relaxed for DM mass in the same range with the same choices of $n$.

So far, we have worked with some discrete values of $\left\{n, T_{R}\right\}$ with $T_{R} \gtrsim 1 \mathrm{GeV}$ and $2 \leq n \leq 6$. The explicit dependence of the DM relic on the fast expansion 


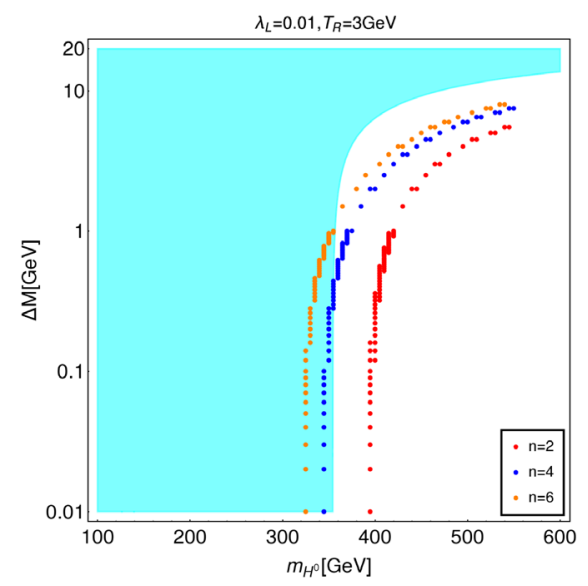

(a)

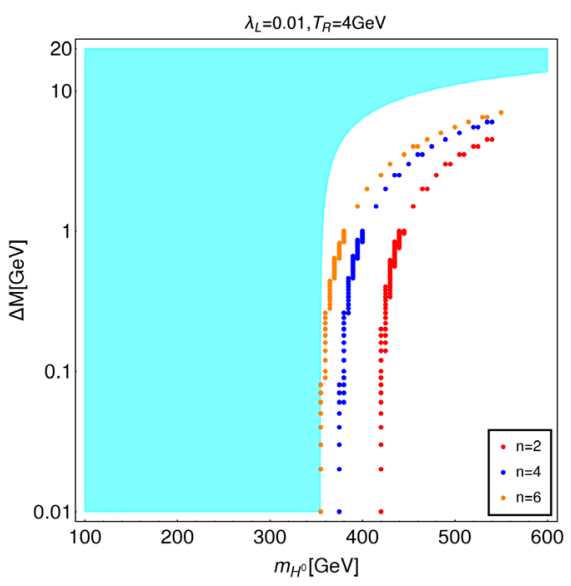

(b)

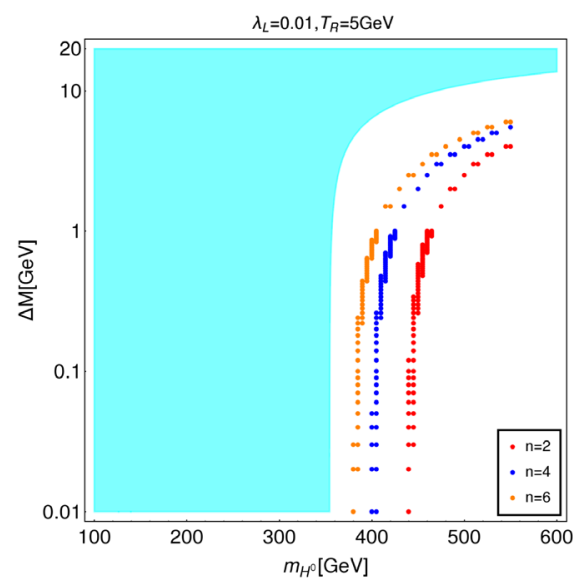

(c)

FIG. 3. Relic satisfied points (red, blue, and orange) are shown in $m_{H^{0}}-\Delta M$ plane as a function of $n$ values considering (a) $T_{R}=3 \mathrm{GeV}$, (b) $T_{R}=4 \mathrm{GeV}$, (c) $T_{R}=5 \mathrm{GeV}$, and a uniform $\lambda_{L}=0.01$ value. The cyan region is forbidden from the indirect search bound to the $W W$ final state.

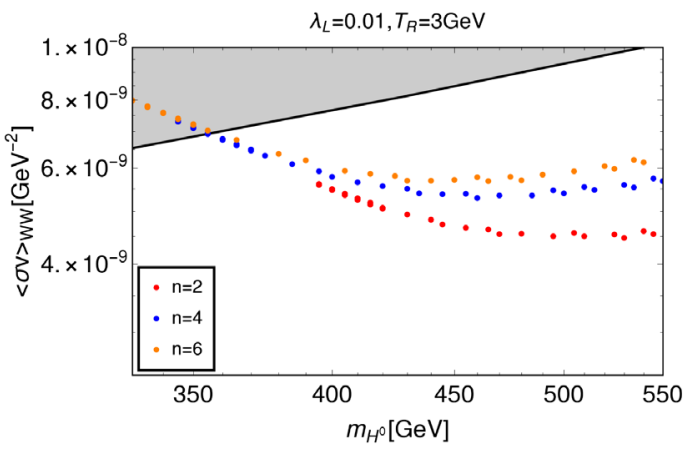

(a)

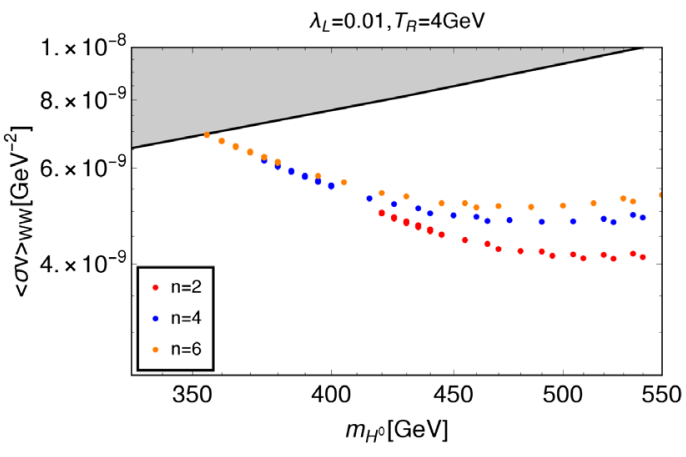

(b)

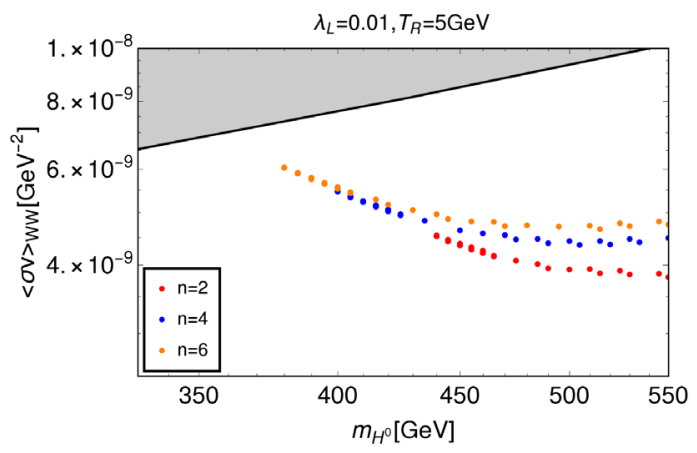

(c)

FIG. 4. Numerical estimate of DM annihilation cross section to $W^{+} W^{-}$final states for the relic satisfied points with different $n$ values as shown in Fig. 3 considering (a) $T_{R}=3 \mathrm{GeV}$, (b) $T_{R}=4 \mathrm{GeV}$, (c) $T_{R}=5 \mathrm{GeV}$, and a uniform $\lambda_{L}=0.01$ value. The black solid line represents the latest bound of nonobservation of the DM at the Fermi experiment.

parameters $n, T_{R}$ are shown in Fig. 6 for a fixed DM mass of $480 \mathrm{GeV}$ considering two different values of $\lambda_{L} \sim 0.01$ and 0.05 . Following the previous scans, here we see a similar trend for $\lambda_{L}=\{0.01,0.05\}$ in the left and right panels, respectively. With the increase in $\Delta M$, we see a smaller $T_{R}$ is required to satisfy the observed relic abundance. This can be understood from the fact that a larger $\Delta M$ leads to underabundance (since DM annihilation dominates over the coannihilation in the given range of $\Delta M)$ and hence a smaller $T_{R}$ is required to trigger a faster expansion following Eq. (7) to satisfy the DM abundance. For the same reason, larger $\Delta M$ requires larger $n$ 's for a fixed $T_{R}$ to produce right relic. Recall that a smaller value of $T_{R}$ for a fixed $n$ (and vice versa) violating the limit in 


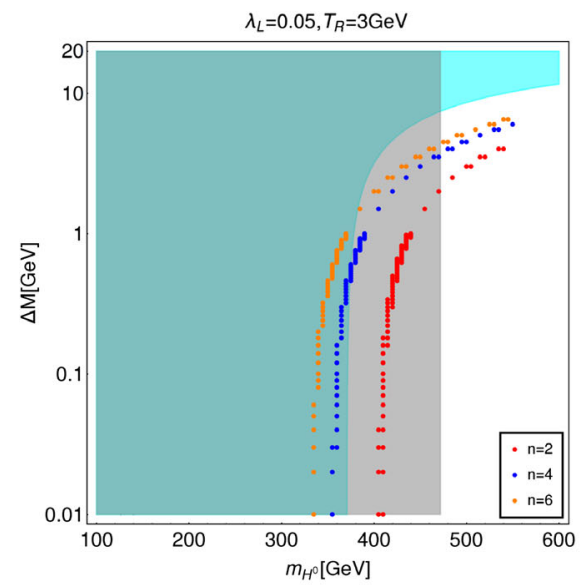

(a)

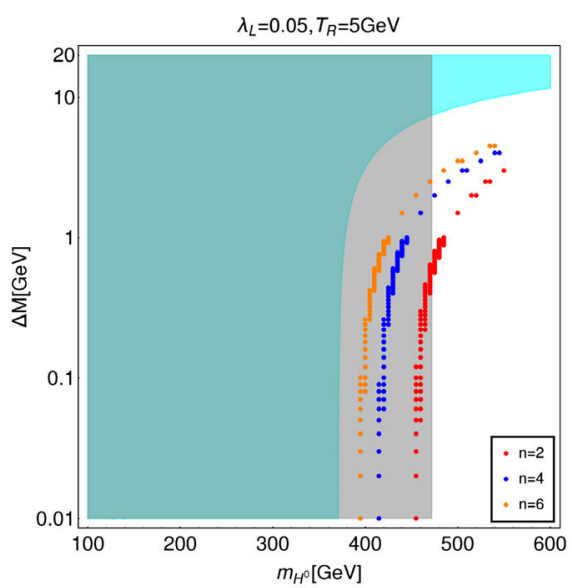

(b)

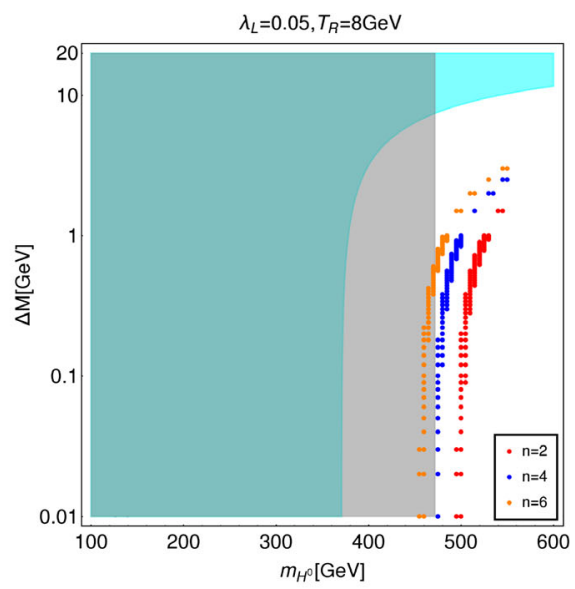

(c)

FIG. 5. Relic satisfied points (red, blue, and orange) are shown in the $m_{H^{0}}-\Delta M$ plane as function of $n$ values considering (a) $T_{R}=3 \mathrm{GeV}$, (b) $T_{R}=5 \mathrm{GeV}$, (c) $T_{R}=8 \mathrm{GeV}$, and a uniform $\lambda_{L}=0.05$ value. The indirect search bound for the $W^{+} W^{-}$final state forbids the cyan region, while the gray shaded region shows the direct search exclusion limit from XENON1T.

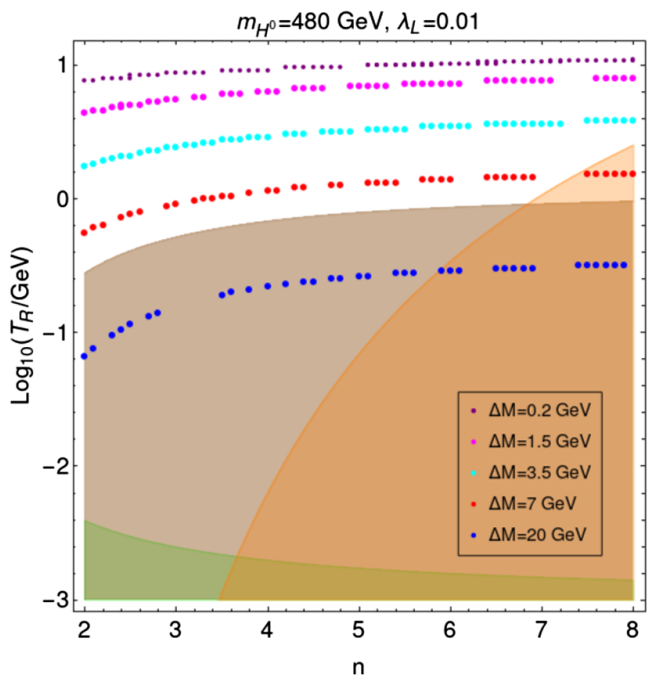

(a)

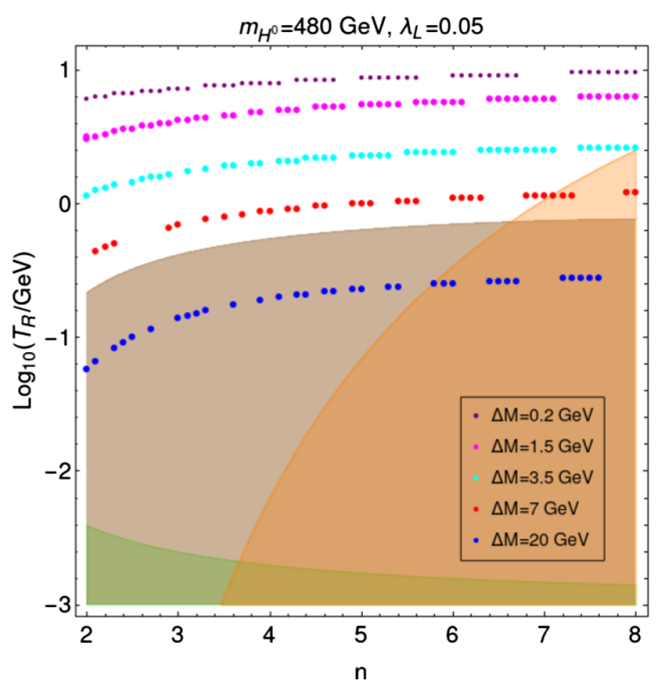

(b)

FIG. 6. Relic satisfied points are shown in the $n-T_{R}$ plane for a fixed DM mass of $480 \mathrm{GeV}$ and different choices of $\Delta M$ for (a) $\lambda_{L}=0.01$ and (b) $\lambda_{L}=0.05$. In both the plots, the green region is forbidden from the BBN bound on $T_{R}$ following Eq. (8), while the orange and the brown regions are disallowed by the nonthermalization of DM above the weak scale [following Eq. (27)] and indirect search constraints, respectively. Any point in the $n-T_{R}$ plane is also subject to an additional constraint arising from the perturbative unitarity bound (discussed later) which is relatively weaker.

Eq. (8) is disfavored from the BBN bound. This $\mathrm{BBN}$-excluded region is shown in either of the plots in green. Larger $\Delta M(\gtrsim 20) \mathrm{GeV}$ regions, as they require smaller $T_{R}$ to satisfy the relic abundance, get discarded from the BBN bound. The brown region indicates the disallowed space by an indirect search constraint, which is also present in Figs. 3 and 5 (shown in cyan), while the orange region is disfavored by the violation of DM thermalization condition before the weak scale following Eqs. (7) and (27).
In principle, a lower bound on $\Delta M$ should also be present in Fig. 6 arising from the condition the heavier mass eigenstates should decay completely before the BBN. However, we find that the obtained bound already lies below our working range of $\Delta M$ as specified earlier and hence does not appear in Fig. 6. We also see that, for fixed $\Delta M$ and $m_{H^{0}}$, larger $\lambda_{L}$ prefers low $T_{R}$ (for a fixed $n$ ) or larger $n$ (for a fixed $T_{R}$ ). This is typically attributed to the DM annihilation cross section that has a quadratic dependence on $\lambda_{L}$. The requirement of thermalization of the DM 


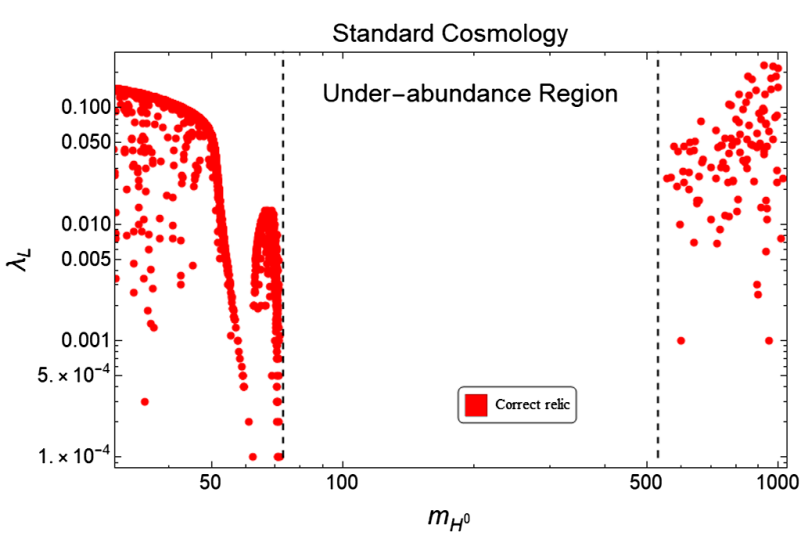

(a)

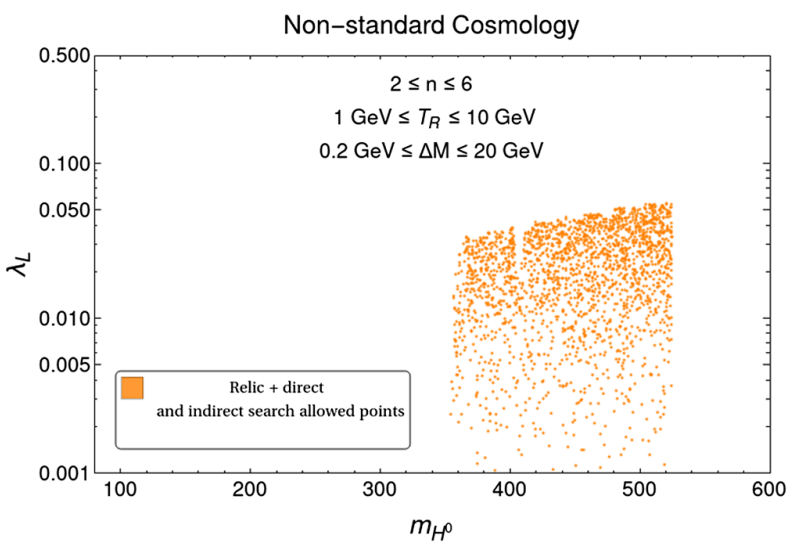

(b)

FIG. 7. Left: the IDM parameter space in $m_{H^{0}}-\lambda_{L}$ plane validated with Planck observed relic density bound considering standard cosmology. The presence of the desert (relic underabundant) region for $80 \mathrm{GeV} \lesssim m_{H^{0}} \lesssim 525 \mathrm{GeV}$ can clearly be seen. Right: same as left but in a nonstandard cosmological background where the desert region has been revived, satisfying all constraints: relic density, direct detection due to XENON1T, and indirect search. The values of the relevant parameters are mentioned in the plot legends.

above the weak scale disallows larger values of $n$ for smaller $T_{R}$ as shown by the orange region. A smaller $T_{R}$ results in a faster expansion, causing the DM to fall out of thermal equilibrium in early times. This can be prohibited by tuning $n$ to smaller values such that the DM thermalizes at temperatures above the weak scale. Thus, larger $n$ values are discarded for smaller $T_{R}$. This bound remains the same for $\lambda_{L}=0.05$ (shown in the right panel), since the DM annihilation is dominantly controlled by the gauge coupling $g_{2}$ as discussed earlier in detail. With these outcomes, it is understandable that the fast expansion parameters are well restricted by all the combined constraints irrespective of the value of $\lambda_{L}$. Finally, it is crucial to note that the indirect search constraint disfavors DM mass less than or approximately equal to $350 \mathrm{GeV}$, immaterial of the choice of $\lambda_{L}$, eliminating the possibility of resurrecting the low DM mass region satisfying all relevant constraints. ${ }^{6}$ This, together with the direct detection bound (important for larger $\lambda_{L}$ ), typically rules out the allowed parameter space for a DM mass of $200 \mathrm{GeV}$ that was overlooked in earlier work [35]. This can further be verified from Fig. 7 where in the left panel we present the allowed points from relic density considering standard cosmology in the $m_{H^{0}}-\lambda_{L}$ plane. It clearly shows the presence of a void (underabundant) in the range $80 \mathrm{GeV} \lesssim m_{H^{0}} \lesssim 525 \mathrm{GeV}$. In the right panel, considering a fast expanding Universe, we perform a random scan for different ranges of the relevant parameters and sort out the points satisfying observed relic abundance, indirect search, and direct search due to XENON1T exclusion. We find viable parameter space in the said mass range under nonstandard scenario satisfying all relevant bounds. Also, nonexistence of any allowed points for $m_{H^{0}} \lesssim 350 \mathrm{GeV}$

\footnotetext{
${ }^{6}$ This implies the desert region for IDM, taking into account the indirect search bound, typically lies in the range $350 \lesssim m_{H^{0}} \lesssim$ $525 \mathrm{GeV}$ for small $\lambda_{L}$.
}

confirms our earlier observations. ${ }^{7}$ From the right panel, one can notice, for a given DM mass, it is possible to choose $\lambda_{L}$ as small as 0.001 . For such a small $\lambda_{L}(\lesssim 0.01)$, the direct search cross section [Eq. (26)] becomes safe from the XENON1T exclusion limit, and an indirect search provides the most stringent bound on DM mass (see Fig. 3). In contrast, for a larger $\lambda_{L} \gtrsim 0.05$, a direct search constraint becomes important (see Fig. 5). The DM annihilation cross section (or, equivalently, the relic abundance), however, is controlled dominantly by the $S U(2)_{L}$ gauge coupling, while $\lambda_{L}$ plays a subdominant role. Therefore, in the present setup, it is possible to work with further lower $\lambda_{L}(\lesssim 0.001)$ satisfying all pertinent bounds, without altering the allowed range of DM mass.

\section{Collider probe of the IDM desert region}

As we have already seen, for the mass region of our interest, satisfying relic abundance and exclusion limits from (in)direct searches, the mass splitting $\Delta M$ can be at best a few GeV for any $n \geq 0$. Such small $\Delta M$ regions are indeed challenging to probe at the colliders. This extremely compressed scenario can be probed with identifying the charged track signal of a long-lived charged scalar, which is $H^{ \pm}$in this case $[66,85]$. For $\Delta M \approx 200 \mathrm{MeV}$, the charged scalar has the dominant decay mode: $H^{ \pm} \rightarrow \pi^{ \pm} H^{0}$. Following Ref. [66], one can analytically obtain the $H^{ \pm} \rightarrow \pi^{ \pm} H^{0}$ decay width in the $\Delta M / m_{H^{ \pm}} \ll 1$ limit as

$$
\Gamma_{H^{ \pm} \rightarrow \pi^{ \pm} H^{0}}=\frac{g_{2}^{4} f_{\pi}^{2}}{64 \pi} \frac{\Delta M^{3}}{m_{W}^{4}} \sqrt{1-\frac{m_{\pi^{ \pm}}^{2}}{\Delta M^{2}}},
$$

\footnotetext{
${ }^{7}$ This lower bound takes into account the thermalization condition.
} 
where $g_{2}$ is the $S U(2)_{L}$ gauge coupling strength, the charged pion mass $m_{\pi^{ \pm}}=139.57 \mathrm{MeV}$, and $f_{\pi} \approx$ $130 \mathrm{MeV}$ is the pion decay constant. Note that the decay width and hence the lifetime $\tau_{H^{ \pm} \rightarrow \pi^{ \pm} H^{0}} \equiv \frac{1}{\Gamma_{H^{ \pm} \rightarrow \pi^{ \pm} H^{0}}}$ of the charged scalar are inversely proportional to the mass splitting. Therefore, a large mass splitting shall produce a charged track of smaller length and vice versa. Depending on $\Delta M$, two scenarios can arise:

(i) For $\Delta M \in\{140-200\} \mathrm{MeV}, H^{ \pm}$shall give rise to disappearing charged track of length $L=$ $c \tau \simeq \mathcal{O}(100-10) \mathrm{cm}$ with branching ratio (of $\left.H^{ \pm} \rightarrow \pi^{ \pm} H^{0}\right)$ close to $100 \%$. For $\Delta M>$ $200 \mathrm{MeV}$, the branching ratio gets reduced as new decay modes start to appear.

(ii) For $\Delta M<m_{\pi}$, the decay is defined via the threebody process $H^{ \pm} \rightarrow W^{\star} H^{0} \rightarrow \ell \nu_{\ell} H^{0}$, which is proportional to $\Delta M^{5} / m_{W}^{4}$. The decay width of such a process turns out to be less than or approximately equal to $10^{-18} \mathrm{GeV}$, resulting in a decay length of $c \tau \gtrsim \mathcal{O}(\mathrm{m})$, implying $H^{ \pm}$remains stable at collider scales and decay outside the detector, giving rise to a stable charged track.

We have used CalcHEP [86] to compute the decay width (total and partial) numerically taking care of both the twobody and three-body decay of $H^{ \pm}$.

A disappearing track results from the decay products of a charged particle which go undetected because they either have too small momentum to be reconstructed or have interaction strength such that they do not produce hits in the tracker and do not deposit significant energy in the calorimeters. Searches for disappearing track signatures have been performed both by CMS $[48,87]$ and ATLAS [88] in the context of supersymmetry for a center-of-mass energy of $\sqrt{s}=13 \mathrm{TeV}$, setting upper limits on the chargino mass and production cross section. To recast the exact limits from CMS and ATLAS, one has to perform a careful re- construction and selection of events by employing suitable cuts taking into account the generator-level eciency along with the background estimation. This is beyond the scope of this paper. ${ }^{8}$ Alternatively, here, we make an estimate of the lifetime of $H^{ \pm}$with the allowed values of $\Delta M$ and $M_{H^{0}}$ and project the available limits from CMS [87] to realize if it is at all feasible to see the charged tracks in colliders. This, in turn, could imply a collider probe for an alternative cosmological history of the Universe.

As stated earlier, for $\Delta M \in\{140-200\} \mathrm{MeV}, H^{ \pm}$ decays dominantly into the $\pi^{ \pm}, H^{0}$ final state, while for $\Delta M<m_{\pi}$, the decay turns out to be the semileptonic threebody final state. In the top left panel of Fig. 8, we see a manifestation of this, where the branching $\operatorname{Br}\left(H^{ \pm} \rightarrow\right.$ $\pi^{ \pm}, H^{0}$ ) into the pion final state decreases with the increase in $\Delta M$ as the three-body decay starts dominating. Note

\footnotetext{
${ }^{8} \mathrm{~A}$ recent analysis can be found in Ref. [89].
}

that, in this case, the DM mass also varies in the range $m_{H^{0}} \in\{450-463\} \mathrm{GeV}$. Following Eq. (28), we also expect, for large $\Delta M$, the lifetime $\tau_{H^{ \pm} \rightarrow \pi^{ \pm} H^{0}}$ should decrease, producing a shorter disappearing track. This is exactly what we see in the top right panel of Fig. 8. Thus, a larger $\operatorname{Br}\left(H^{ \pm} \rightarrow \pi^{ \pm}, H^{0}\right)$ implies a longer lifetime $\tau_{H^{ \pm} \rightarrow \pi^{ \pm} H^{0}}$ (and a smaller $\Delta M$ ) or equivalently a longer track length. This, in turn, places constraints on the model parameter, which we are going to discuss next. One should also note the presence of points satisfying relic abundance for $n=2$ with different choices of $T_{R}$ on the same plane, indicating the possibility of testing benchmark points obtained from the analysis in the last sections in collider experiments.

In the bottom left panel of Fig. 8, we project the experimental limit $[48,87]$ from CMS on the decay lifetime of $H^{ \pm}$obtained using our model parameters. The red line corresponds to the CMS limit where the decaying charged particle has $100 \%$ decay branching fraction into pion final state, whereas for the blue line, the pion decay branching fraction is $95.5 \%$. The black thick curve shows the total lifetime of $H^{ \pm}$as a function of $m_{H^{ \pm}}$obtained numerically for a fixed DM mass of $450 \mathrm{GeV}$. We again show three benchmark points where observed relic density can be obtained for $n=2$ with different $T_{R}$. We note, based on the approximate analysis, that $\Delta \mathrm{M} \lesssim 200 \mathrm{MeV}$ is tightly constrained from CMS and likely to be ruled out, which also agrees with earlier observations [66]. However, large $\Delta M(>200 \mathrm{MeV})$ regions with shorter lifetime (for example, the point denoted by $\mathrm{X}$ in the bottom left panel of Fig. 8) still can be seen lying beyond the reach of present CMS bound. It is understandable that, by tuning $n, T_{R}$, it is always possible to accommodate points for $\Delta M>$ $200 \mathrm{MeV}$ which satisfy relic density that are safe from CMS exclusion. We can thus infer, for any given $\left(n, T_{R}\right)$, the region of parameter space satisfying DM constraints with lifetime less than or approximately equal to $0.1 \mathrm{~ns}$ (equivalent to a track length of less than or approximately equal to $1 \mathrm{~cm}$ ) is beyond the present sensitivity of the CMS experiment, and thus safe. Finally, in the bottom right panel, we show the production cross section for the processes $p p \rightarrow H^{+} H^{-}, H^{ \pm} H^{0}\left(A^{0}\right)$ at $\sqrt{s}=13 \mathrm{TeV}$. A detailed analysis utilizing the numerically obtained production cross section can constrain $m_{H^{ \pm}}$and therefore the DM mass, by providing the number of disappearing track events for a given luminosity. However, here, we only show that our model parameters can give rise to a sizeable production cross section in colliders abiding all DM constraints. For computing the production cross section, we have again relied upon CalcHEP [86] and used CTEQ61 as the representative parton distribution function [90]. We see, for DM mass greater than or approximately equal to $400 \mathrm{GeV}$, the production cross section is approximately $2 \mathrm{fb}$. For all the plots, to show the corresponding DM parameter space, we have chosen $\lambda_{L}=0.01$ such that the 


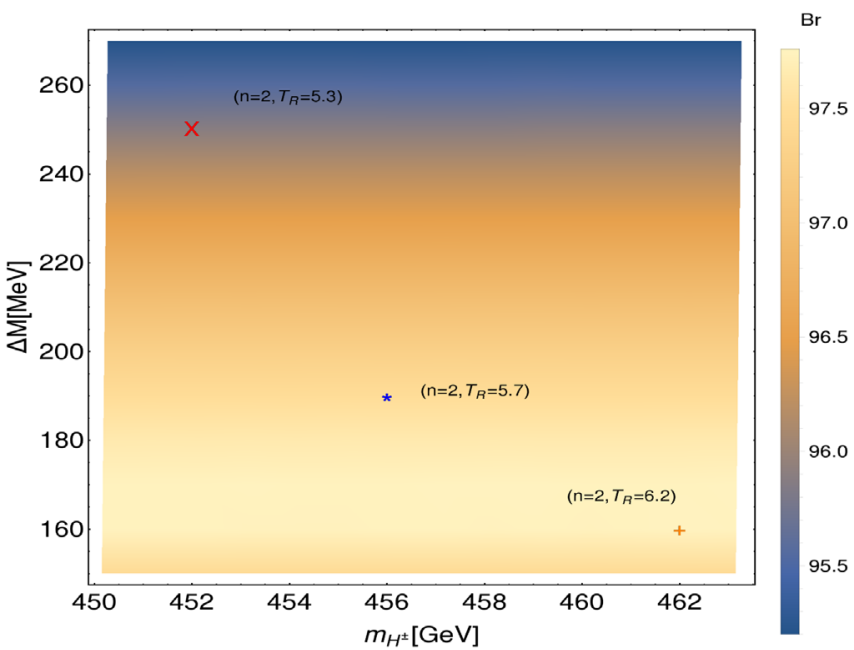

(a)

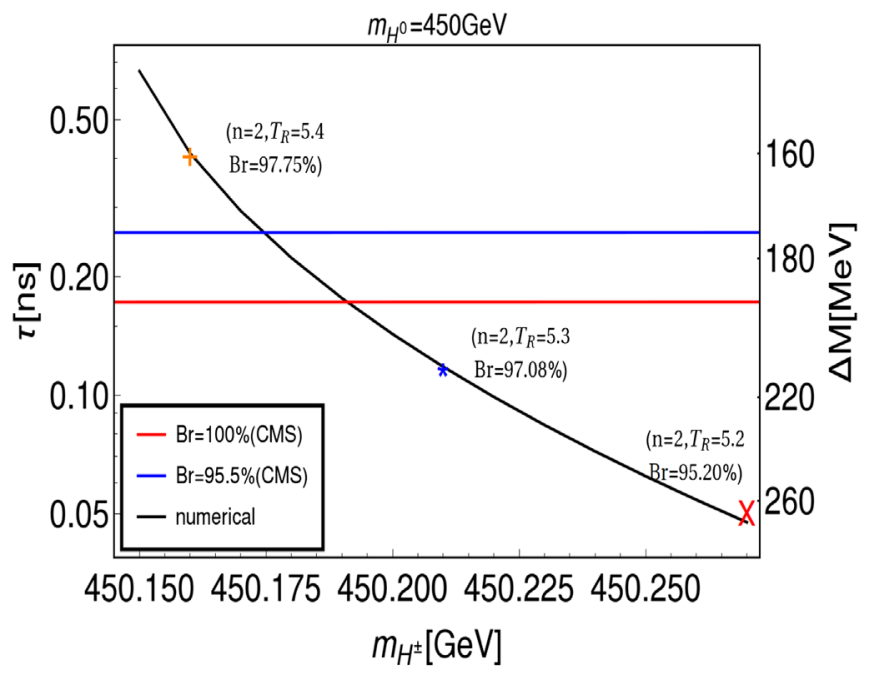

(c)

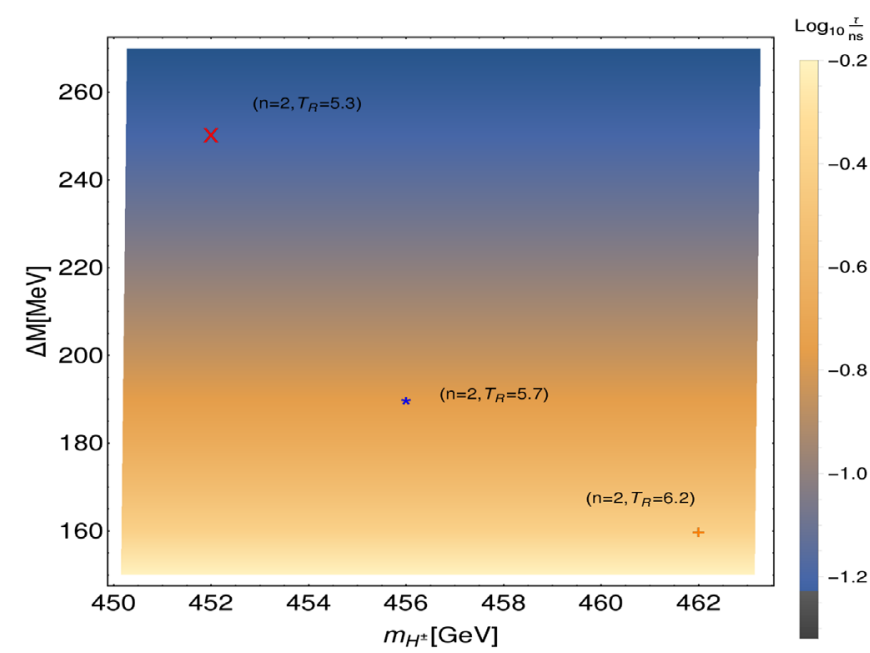

(b)

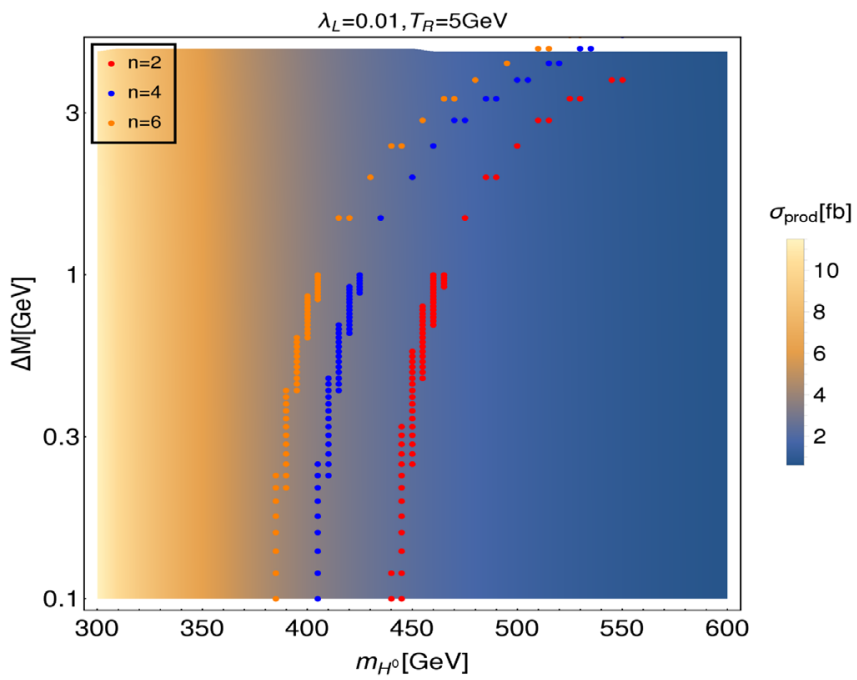

(d)

FIG. 8. Top left: variation of decay branching ratio for $H^{ \pm}$in the bidimensional plane of $m_{H^{ \pm}}-\Delta M$ where the relic density satisfied benchmark points are denoted by $\mathrm{X}, \star$, and + for $n=2$ and different choices of $T_{R}$ in GeV. Top right: same as top left, but the variation is shown against the lifetime $\tau$ (in nanoseconds) of $H^{ \pm}$decay. Bottom left: total decay lifetime of $H^{ \pm}$as a function of $m_{H^{ \pm}}$where the relic satisfied points are marked by $\mathrm{X}$, $\star$, and + for $n=2$ and different choices of $T_{R}$ (in $\left.\mathrm{GeV}\right)$. On the same plane, we also show exclusion limits from CMS at $\sqrt{s}=13 \mathrm{TeV}$ corresponding to the $100 \%$ (in red) and $95.5 \%$ (in blue) branching fraction (see the text for details). Bottom right: variation of production cross section for $p p \rightarrow H^{+} H^{-}, H^{ \pm} H^{0}\left(A^{0}\right)$ at $\sqrt{s}=13 \mathrm{TeV}$ in the $\Delta M-m_{H^{0}}$ plane where the color bar represents the production cross section in units of $f b$. On the same plane, we show the dark matter relic density satisfied points for $n=2,4,6$ as indicated respectively by red, blue, and orange, considering $T_{R}=5 \mathrm{GeV}$.

DM is safe from direct and indirect search constraints. We conclude this section by observing that a charged track of length less than or approximately equal to $\mathcal{O}(1)$ could indeed be a probe for a nonstandard cosmological parameters for the IDM providing evidence for fast expanding pre-BBN era at the LHC.

\section{B. ITDM in a fast expanding Universe}

As mentioned in the beginning, in order to recover the desert region beyond the IDM paradigm, we also apply the prescription of the modified Hubble rate due to fast expansion to scalar DM with larger representation under $S U(2)_{L}$. Here, we describe the general structure of a $S U(2)_{L}$ triplet dark matter model. In this setup, the $S M$ is extended by introducing a $S U(2)_{L}$ triplet scalar with hypercharge $Y=0$. An additional $Z_{2}$ symmetry is also imposed under which the triplet transforms nontrivially. It is also considered that the triplet has zero $\mathrm{VEV}$. The scalar potential under $\mathrm{SM} \times Z_{2}$ symmetry then reads [50] 


$$
\begin{aligned}
V(H, T) & \supset \mu_{H}^{2}|H|^{2}+\lambda_{H}|H|^{4}+\frac{\mu_{T}^{2}}{2} \operatorname{Tr}\left[T^{2}\right] \\
& +\frac{\lambda_{T}}{4 !}\left(\operatorname{Tr}\left[T^{2}\right]\right)^{2}+\frac{\lambda_{H T}}{2}|H|^{2} \operatorname{Tr}\left[T^{2}\right],
\end{aligned}
$$

where $H$ is the SM-like Higgs doublet and the triplet $T$ is parametrized as

$$
T=\left(\begin{array}{cc}
T^{0} / \sqrt{2} & -T^{+} \\
-T^{-} & -T^{0} / \sqrt{2}
\end{array}\right) .
$$

Now, after electroweak symmetry breaking, the masses of the physical scalar triplets are given by

$$
m_{T^{0}, T^{ \pm}}^{2}=\mu_{T}^{2}+\frac{\lambda H T}{2} v^{2},
$$

with $v=246 \mathrm{GeV}$. Notice that, although the mass of neutral and charged triplet scalar are degenerate [Eq. (31)], a small mass difference $\delta m \simeq 166 \mathrm{MeV}$ can be generated via one-loop radiative correction [91] that makes $T^{0}$ as the lighter component and hence a stable DM candidate. This is the crucial difference between IDM and scalar triplet DM where in IDM the mass difference is a free parameter while for scalar triplet this is fixed from one-loop correction. The conditions bounded from below for the scalar potential in all field directions in Eq. (30) require

$$
\lambda_{H, T} \geq 0 ; \quad \sqrt{\lambda_{H} \lambda_{T}}>\frac{1}{2}\left|\lambda_{H T}\right| .
$$

Apart from the theoretical constraints arising from the stability, perturbativity, and tree-level unitarity of the scalar potential, one needs to also consider the experimental constraints on the parameters of the scalar potential. As the charged and neutral components of the triplet scalar are almost degenerate, the contributions to the $T$ and $U$ parameters are very much suppressed in this scenario. However, the charged component $T^{ \pm}$can contribute significantly to the Higgs diphoton signal strength, which is accurately measured $\mu_{\gamma \gamma}=0.99 \pm 0.14$ from ATLAS [92] and $\mu_{\gamma \gamma}=1.17 \pm 0.10$ from CMS. It has recently been shown $[93,94]$ that searches for disappearing tracks at the LHC excludes a real triplet scalar lighter than $287 \mathrm{GeV}$ using $36 \mathrm{fb}^{-1}$ of data at $\sqrt{s}=13 \mathrm{TeV}$.

We again numerically solve the BEQ in Eq. (22) with the modified Hubble rate in Eq. (7) and determine the subsequent DM relic density for different choices of the fast expansion parameters $n, T_{R}$. In the top and middle panels, of Fig. 9, we show the variation of the DM relic abundance as a function of the ITDM mass. Here, we have kept the portal coupling fixed and obtained the resulting direct and indirect search exclusion regions for $\lambda_{H T}=0.01$. The parameter space excluded by the XENON1T limit is shown by the brown region where the direct search cross section is given by [42]

$$
\sigma_{n-T^{0}}^{\mathrm{SI}}=\frac{\lambda_{H T}^{2} f_{N}^{2}}{4 \pi} \frac{\mu^{2} m_{n}^{2}}{m_{h}^{4} m_{T^{0}}^{2}}
$$

while the indirect search exclusion due to the $W^{+} W^{-}$final state is shown by the cyan region. Since the mass splitting $\delta m$ is no longer a free parameter and fixed to a small value of $\delta m \simeq 166 \mathrm{MeV}$, coannihilation plays the dominating role here. As a result, the right relic is obtained in the case of ITDM for a very large DM mass $m_{T^{0}} \sim 1.8 \mathrm{TeV}$ as shown by the red curve $(n=0)$ in each plot. Once fast expansion is introduced, there is drastic improvement in the parameter space. As one can see, for $T_{R}=1 \mathrm{GeV}$, the right relic density is achievable for $m_{T^{0}} \sim 800 \mathrm{GeV}$ with $n=2$ (blue curve), while for $T_{R}=2 \mathrm{GeV}$, the relic satisfied mass is around $900 \mathrm{GeV}$ with $n=2$. As inferred earlier, this happens because for smaller $T_{R}$ the expansion rate increases following Eq. (7). This is being compensated by a smaller choice of the DM mass to satisfy the observed abundance since $\langle\sigma v\rangle \propto 1 / m_{T^{0}}^{2}$. Enhancement of $n$ could provide further smaller relic satisfied DM mass consistent with direct, indirect, and LHC searches. Varying $\lambda_{H T}$ would give similar results since the effective annihilation cross section is mostly dominated by gauged-mediated coannihilation, hence almost insensitive to $\lambda_{H T}$ unless it is very large (greater than or approximately equal to 0.1 ,) which is anyway disfavored from direct and indirect search bounds. In the bottom panel of Fig. 9, we vary the DM mass $m_{T^{0}}$ by keeping $\lambda_{H T}=0.01$ and obtain the resulting relic abundance allowed parameter space in the $T_{R}-m_{T^{0}}$ plane for different choices of $n$. Here, we again see the manifestation of faster expansion elaborated above; i.e., for a fixed DM mass, a smaller $n$ (in red) needs a smaller $T_{R}$ in order to obtain the observed relic density. Note that in all cases, we have considered $T_{R} \geq 1 \mathrm{GeV}$ to ensure that ITDM remains in thermal equilibrium at high temperature. Limits from direct, indirect, and LHC searches are also projected with the same color code as before. Taking all relevant constraints into account, we see from bottom panel of Fig. 9 that the region $m_{T^{0}} \gtrsim 450 \mathrm{GeV}$ can be recovered considering $2 \leq n \leq 6$ and $T_{R} \gtrsim 1 \mathrm{GeV}$. We find it is also possible to resurrect part of the parameter space below $450 \mathrm{GeV}$ for $T_{R}<1 \mathrm{GeV}$ ensuring the DM thermalizes in the early Universe depending on the choice of $n$. This is, however, in contrast to the case of IDM dark matter, where the lower bound on the allowed DM mass (greater than or approximately equal to $350 \mathrm{GeV}$ ), satisfying thermalization criteria, is almost independent of the fast expanding parameters.

The discovery prospects for a real triplet extension of the SM at the colliders are discussed in Refs. [93,94]. As inferred in Ref. [93], the $13 \mathrm{TeV}$ LHC excludes a real triplet lighter than $\{287,608,761\} \mathrm{GeV}$ for $\mathcal{L}=\{36,300,3000\} \mathrm{fb}^{-1}$ of luminosity. The present case 


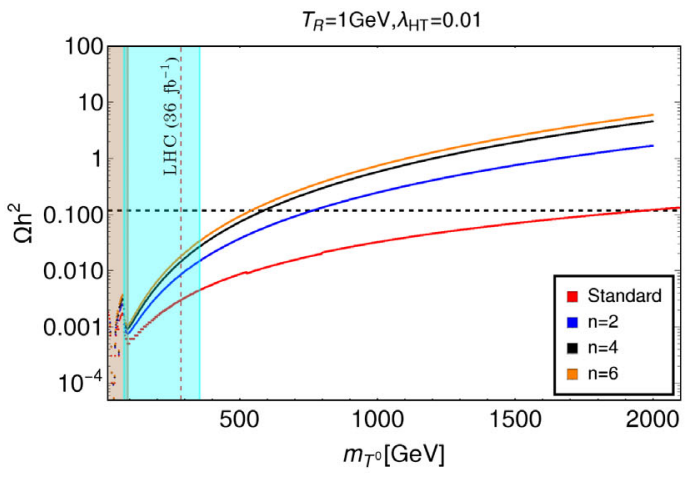

(a)

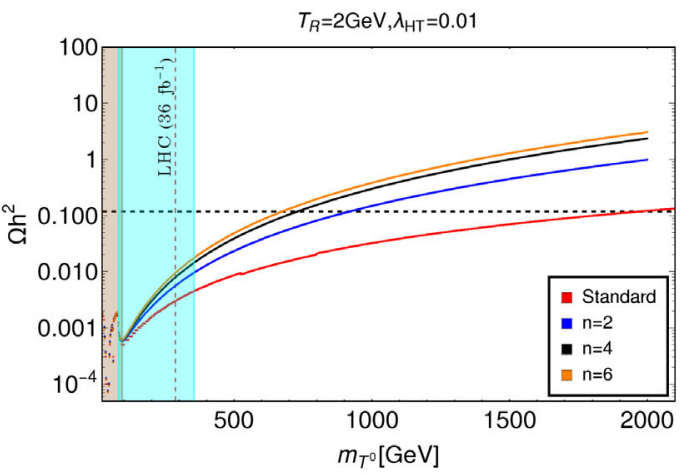

(b)

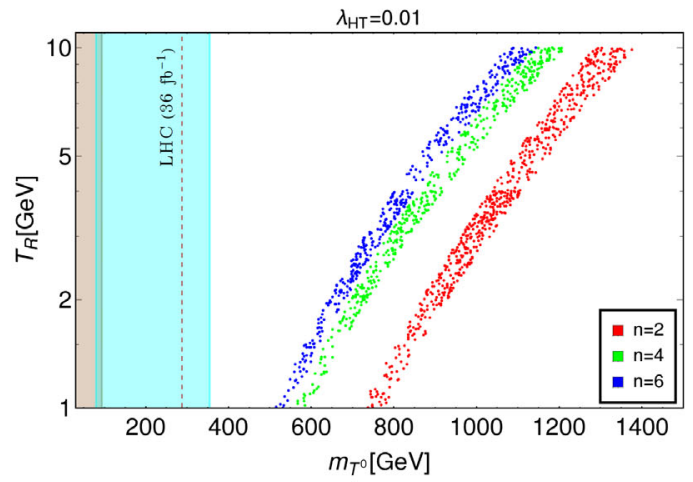

(c)

FIG. 9. Top: variation of DM relic abundance with ITDM mass where the colorful curves correspond to different values of $n$ for a fixed $T_{R}$ as mentioned in the plot legends. Bottom: relic density allowed parameter space in the $T_{R}-m_{T^{0}}$ plane for different choices of $n=2$, 4, 6 shown in, respectively, red, green, and blue. The brown and the cyan regions, respectively, show the DM mass region disallowed by the direct search (XENON1T) and indirect search $\left(W^{+} W^{-}\right.$final state) data. The red dashed straight line in each plot shows the limit from LHC on triplet mass for $36 \mathrm{fb}^{-1}$ of luminosity at $\sqrt{s}=13 \mathrm{TeV}$. In all cases, we have set the portal coupling to a fixed value of $\lambda_{H T}=0.01$.

where the neutral triplet scalar is stable and contributes to the DM Universe (ITDM) can be probed at the colliders via disappearing track signature through the decay of the longlived charged component: $T^{ \pm} \rightarrow \pi^{ \pm} T^{0}$ due to small mass splitting $\delta m$. The situation very similar to that in the case of IDM dark matter discussed in Sec. III A 4; hence, we do not further repeat it here.

The requirement of perturbative unitarity of the DM annihilation cross section can forbid some part of the relic density allowed parameter space depending on the choice of $\left\{n, T_{R}\right\}$ [27], thus providing a bound on the DM mass. A general prescription for obtaining the upper bound on thermal dark matter mass using such partial wave unitarity analysis has been worked out in Ref. [95]. The upper limit on the thermally averaged DM interaction cross section is provided by [95]

$$
\langle\sigma v\rangle_{\max } \lesssim \frac{4 \pi}{m_{\mathrm{DM}}^{2}} \sqrt{\frac{x_{f}}{\pi}}
$$

By using the approximate analytical estimate of DM yield in Eq. (23) (following the approach in Ref. [77]), the freeze-out temperature $x_{f}$ can be approximately determined by using the semianalytical expression for DM yield by equating DM abundances before and after freeze-out [see Eq. (A2)],

$$
e^{x_{f}} x_{f}^{1 / 2} \simeq \frac{c(c+2)}{c+1} \times \frac{0.192 M_{\mathrm{pl}}}{g_{*}^{1 / 2}} \frac{\langle\sigma v\rangle m_{\mathrm{DM}}}{\left(\frac{x_{r}}{x}\right)^{n / 2}}
$$

with $c \sim \mathcal{O}(1)$ constant. We calculate the annihilation cross section that gives rise to the right relic abundance numerically and compare that with the maximum cross section allowed by the partial wave unitarity. This eliminates a part of the parameter space for a fixed DM interaction rate, as shown by the purple region in Fig. 10. It turns out that for both IDM [43] and ITDM [96] the leading contribution to the DM annihilation cross section is $s$-wave dominated. We find that regions with large $n \geq 2$ (and small $T_{R}$ ) are typically in tension with the unitarity bound at the higher range of DM mass. This is expected, since for large $n$ (or small $T_{R}$ ) the Hubble parameter is large, and hence the interaction rate needs to be larger to avoid overabundance. This is in conflict with the maximum allowed annihilation 


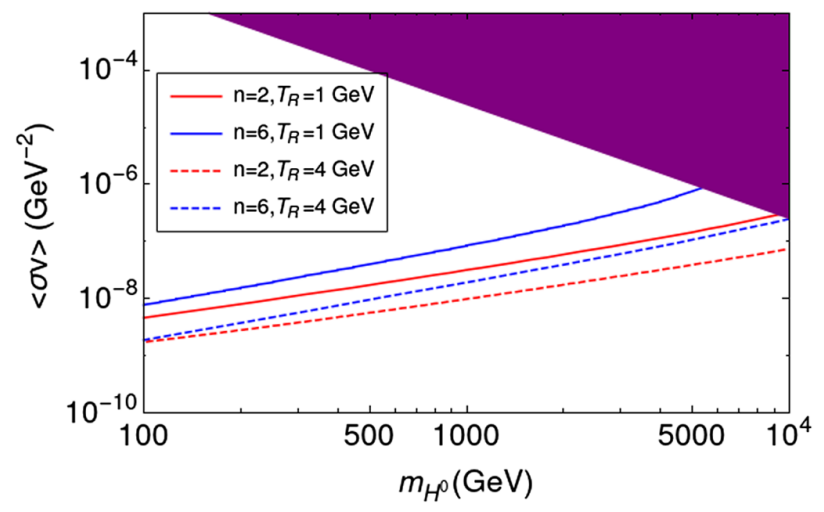

FIG. 10. Required order of cross sections to satisfy the observed DM abundance for different choices of $\left\{n, T_{R}\right\}$ are shown as a function of DM mass. The purple region is disfavored by the perturbative unitarity bound on the DM pair annihilation cross section (see the text for details).

cross section, disfavoring large $\langle\sigma v\rangle$. Now, for the case of IDM, we are specifically interested in the mass window $m_{W} \lesssim m_{H^{0}} \lesssim 525 \mathrm{GeV}$, while for ITDM, $m_{T^{0}} \lesssim 2 \mathrm{TeV}$. On the other hand, as explained earlier, we choose $n \leq 6$ $\left(T_{R} \gtrsim 1 \mathrm{GeV}\right)$ to ensure that the DM thermalizes in the early Universe above the weak scale. Thus, within our working regime of $n$ and $T_{R}$, we find the partial wave unitarity bound does not pose any serious constraint for the DM mass range of our interest.

\section{CONCLUSION}

In this work, considering a form of alternative cosmology, we revisit two popular DM scenarios where the DM is part of $S U(2)_{L}$ multiplets (other than singlet). We first take up the minimal inert doublet model, in which it is observed that an intermediate DM mass range $80 \mathrm{GeV} \lesssim m_{\mathrm{DM}} \lesssim$ $525 \mathrm{GeV}$ is disfavored in a radiation-dominated Universe due to relic underabundance via freeze-out. In an attempt to circumvent this, extension of the minimal inert doublet model or existence of multiple DM candidates has been proposed earlier. Here, we follow a different route and find that, without resorting to an extended particle spectrum, revival of the desert region is possible in the presence of a nonstandard epoch in the early Universe. We obtain the parameter space accounting for the correct relic abundance for single-component inert doublet DM by varying the relevant parameters responsible for the fast expansion of the Universe. Subsequently, we see that a major part of the relic density allowed region gets ruled out from DM direct and indirect search constraints and this in turn puts a restriction on the fast expansion parameters. In particular, we found that for $\lambda_{L}=0.01$ the DM mass below $350 \mathrm{GeV}$ is ruled out irrespective of the cosmological history of the early Universe. The bound turns severe for larger $\lambda_{L}$, i.e., for a higher interaction rate. While for pure IDM bounds from relic density and (in)direct search experiments do not allow a large mass splitting, for the inert scalar triplet, on the other hand, this happens naturally due to small radiative mass splitting. We then discuss possible collider signature for pure IDM under the influence of fast expansion and find that the newly obtained parameter space can be probed via the identification of the charged track signal of a long-lived charged scalar. The resulting track length depends on the mass splitting between the charged and neutral component of the inert scalar doublet. The track length [less than or approximately equal to $\mathcal{O}(1) \mathrm{cm}$ ] for such a long-lived scalar, however, is below the sensitivity from the present CMS/ATLAS search and hence leaves open the possibility of being probed in future experiments. This also implies the prospect of probing the modified cosmological history of the Universe in collider experiments.

We extend our analysis by applying the same methodology to scrutinize the case for hyperchargeless real triplet scalar DM, anticipating such a modification in DM parameter space should also be observed for larger representation of the DM field. We show that a significant parameter space $\left(m_{T^{0}} \gtrsim 450 \mathrm{GeV}\right.$ considering $2 \leq n \leq 6$ and $T_{R} \gtrsim 1 \mathrm{GeV}$ ) satisfying the relic density and other DM search bounds for $m_{T^{0}} \lesssim 2 \mathrm{TeV}$ and portal coupling $\lambda_{H T}=0.01$ can indeed be restored for the scalar triplet scenario, which is otherwise disallowed. We thus conclude that this prescription can be applied for any DM candidate which is a part of a $S U(N)$ multiplet or even for different multicomponent DM frameworks. Implications of our analysis on different aspects of particle physics and cosmology such as electroweak phase transitions, prediction of gravitational waves, neutrino physics, and leptogenesis remain open. We keep these studies for future endeavors.

\section{ACKNOWLEDGMENTS}

One of the authors, A. K. S. appreciates Sudipta Show for several discussions during the course of work. A. K. S. is supported by NPDF Grant No. PDF/2020/000797 from Science and Engineering Research Board, Government of India. P. G. would like to acknowledge the support from DAE, India for the Regional Centre for Accelerator based Particle Physics (RECAPP), Harish Chandra Research Institute. F. S. Q. is supported by the Sao Paulo Research Foundation (FAPESP) through Grant No. 2015/15897-1 and ICTP-SAIFR FAPESP Grant No. 2016/01343-7. F. S. Q. acknowledges support from CNPq Grants No. 303817/ 2018-6 and No. 421952/2018-0 and the Serrapilheira Institute (Grant No. Serra-1912-31613).

\section{APPENDIX A: SEMIANALYTICAL FREEZE-OUT YIELD}

To obtain a semianalytical expression for the DM yield under the influence of fast expansion, we closely follow Ref. [27]. Assuming the DM freezes out during the epoch 
of $\eta$ domination, i.e., $x_{f} \ll x_{r}$, the BEQ in Eq. (22) can be approximated as

$$
\frac{d Y_{\mathrm{DM}}}{d x} \simeq-A \frac{\langle\sigma v\rangle}{x^{2-n / 2} x_{r}^{n / 2}}\left(Y_{\mathrm{DM}}^{2}-Y_{\mathrm{DM}}^{\mathrm{eq}^{2}}\right)
$$

with $A=\frac{2 \sqrt{2} \pi}{3 \sqrt{5}} \sqrt{g_{*}} m_{\mathrm{DM}} M_{\mathrm{pl}}$. Defining $\Delta \equiv Y_{\mathrm{DM}}-Y_{\mathrm{DM}}^{\mathrm{eq}}$ and ignoring terms proportional to $\mathcal{O}\left[\Delta^{2}\right]$ in times much earlier than freeze-out (as departure from equilibrium is minimal), while neglecting the equilibrium distribution in the post-freeze-out regime, we obtain

$Y_{\mathrm{DM}}(x) \simeq \begin{cases}Y_{\mathrm{DM}}^{\mathrm{eq}}(x)+\frac{x^{2-n / 2} x_{r}^{n / 2}}{2 A\langle\sigma\rangle} & \text { for } 1<x<x_{f} \\ \left(\frac{1}{Y_{\mathrm{DM}}\left(x_{f}\right)}+A \xi(x)\right)^{-1} & \text { for } x_{f}<x<x_{r},\end{cases}$

where

$$
\xi(x)=\frac{1}{x_{r}^{n / 2}} \int_{x_{f}}^{x} d x \frac{\langle\sigma v\rangle}{x^{2-n / 2}} .
$$

Now, one can expand the thermally averaged cross section in terms of the partial waves as $\langle\sigma v\rangle \simeq \sigma_{s}+$ $\sigma_{p} / x+\mathcal{O}\left(x^{-2}\right)$. Considering $s$-wave domination and on substitution in Eq. (A3), we find

$$
\xi(x)=\frac{\sigma_{s}}{x_{r}^{n / 2}} \begin{cases}\frac{x_{f}^{n / 2-1}-x^{n / 2-1}}{1-n / 2} & \text { for } n \neq 2 \\ \log \left[\frac{x}{x_{f}}\right] & \text { for } n=2 .\end{cases}
$$

After the end of fast expansion regime $\left(x>x_{r}\right)$, the radiation dominates the energy density, and the resulting DM yield reads

$Y_{\mathrm{DM}}(x) \simeq\left(\frac{1}{Y_{\mathrm{DM}}\left(x_{f}\right)}+A \xi_{\mathrm{rad}}(x)\right)^{-1}, \quad x>x_{r}$,

where

$$
\xi_{\mathrm{rad}}(x)=\int_{x_{r}}^{x} d x \frac{\langle\sigma v\rangle}{x^{2}}
$$

\section{APPENDIX B: BBN CONSTRAINTS}

The effect of the new species $\eta$ can be parametrized by an effective number of relativistic degrees of freedom (DOF) as evident from Eq. (5),

$$
\rho(T)=\frac{\pi^{2}}{30} g_{* \mathrm{eff}} T^{4},
$$

with

$$
\begin{aligned}
g_{* \mathrm{eff}} & =g_{*}^{\mathrm{SM}}+\Delta g_{*}^{\eta} \\
& =\left(2+\frac{7}{8} \times 4\right)+\left(2 \times \frac{7}{8} \times N_{\nu}\right)+\left(2 \times \frac{7}{8} \times \Delta N_{\nu}\right),
\end{aligned}
$$

The first two terms in the last equation stand for the SM contribution with the $N_{\nu}$ indicating the number of effective neutrinos. The notation $\Delta N_{\nu}$ accounts for the $\eta$ contribution to the number of relativistic degrees of freedom as obtained from Eq. (5),

$$
\Delta N_{\nu}=\frac{4}{7} g_{*}\left(T_{R}\right)\left(\frac{g_{* s}(T)}{g_{* S}\left(T_{R}\right)}\right)^{(4+n) / 3}\left(\frac{T}{T_{R}}\right)^{n} .
$$

Considering $T_{R}$ around $T_{\mathrm{BBN}}$ and $T \sim T_{\mathrm{BBN}}$, we can assume $g_{* s}(T) \sim g_{* s}\left(T_{R}\right)$. We also use $g_{*}\left(T_{R}\right)=$ $\left(2+\frac{7}{8} \times 4+\frac{7}{8} \times 2 \times 3\right)$ to include the contributions of photon, positrons, and neutrinos and reach

$$
\Delta N_{\nu} \simeq 6.14\left(\frac{T}{T_{R}}\right)^{n}
$$

Since the additional contribution to $N_{\nu}$ is positive, we use the bound $N_{\nu}+\Delta N_{\nu} \lesssim 3.4$ [97] at 95\% C.L. $(2 \sigma)$ and $T \simeq 1 \mathrm{MeV}$ to obtain

$$
T_{R} \gtrsim(15.4)^{1 / n} \mathrm{MeV} \text {. }
$$

[1] D. J. Chung, E. W. Kolb, and A. Riotto, Production of massive particles during reheating, Phys. Rev. D 60, 063504 (1999).

[2] N. Okada and O. Seto, Relic density of dark matter in brane world cosmology, Phys. Rev. D 70, 083531 (2004).
[3] N. Okada and O. Seto, Gravitino dark matter from increased thermal relic particles, Phys. Rev. D 77, 123505 (2008).

[4] N. Okada and S. Okada, Gauss-Bonnet braneworld cosmological effect on relic density of dark matter, Phys. Rev. D 79, 103528 (2009). 
[5] R. Allahverdi and J. K. Osiński, Nonthermal dark matter from modified early matter domination, Phys. Rev. D 99, 083517 (2019).

[6] V. Baules, N. Okada, and S. Okada, Braneworld cosmological effect on freeze-in dark matter density and lifetime frontier, arXiv:1911.05344.

[7] I. R. Waldstein, A. L. Erickcek, and C. Ilie, Quasidecoupled state for dark matter in nonstandard thermal histories, Phys. Rev. D 95, 123531 (2017).

[8] P. Arias, N. Bernal, A. Herrera, and C. Maldonado, Reconstructing non-standard cosmologies with dark matter, J. Cosmol. Astropart. Phys. 10 (2019) 047.

[9] C. Cosme and T. Tenkanen, Spectator dark matter in nonstandard cosmologies, Phys. Rev. D 102, 123534 (2020).

[10] L. Aparicio, M. Cicoli, B. Dutta, F. Muia, and F. Quevedo, Light Higgsino dark matter from non-thermal cosmology, J. High Energy Phys. 11 (2016) 038.

[11] C. Han, Higgsino dark matter in a non-standard history of the universe, Phys. Lett. B 798, 134997 (2019).

[12] M. Drees and F. Hajkarim, Dark matter production in an early matter dominated era, J. Cosmol. Astropart. Phys. 02 (2018) 057.

[13] G. Arcadi, S. Profumo, F. Queiroz, and C. Siqueira, Righthanded neutrino dark matter, neutrino masses, and nonstandard cosmology in a 2HDM, J. Cosmol. Astropart. Phys. 12 (2020) 030.

[14] C. Cosme, M. A. Dutra, T. Ma, Y. Wu, and L. Yang, Neutrino portal to FIMP dark matter with an early matter era, J. High Energy Phys. 03 (2021) 026.

[15] N. A. Bernal, F. Elahi, C. Maldonado, and J. Unwin, Ultraviolet freeze-in and non-standard cosmologies, J. Cosmol. Astropart. Phys. 11 (2019) 026.

[16] R. Allahverdi and J. K. Osiński, Freeze-in production of dark matter prior to early matter domination, Phys. Rev. D 101, 063503 (2020).

[17] C. Maldonado and J. Unwin, Establishing the dark matter relic density in an era of particle decays, J. Cosmol. Astropart. Phys. 06 (2019) 037.

[18] M. Drees and F. Hajkarim, Neutralino dark matter in scenarios with early matter domination, J. High Energy Phys. 12 (2018) 042.

[19] N. Bernal, C. Cosme, and T. Tenkanen, Phenomenology of self-interacting dark matter in a matter-dominated universe, Eur. Phys. J. C 79, 99 (2019).

[20] L. Visinelli, (Non-)thermal production of WIMPs during kination, Symmetry 10, 546 (2018).

[21] A. Arbey, J. Ellis, F. Mahmoudi, and G. Robbins, Dark matter casts light on the early universe, J. High Energy Phys. 10 (2018) 132.

[22] D. Berger, S. Ipek, T. M. Tait, and M. Waterbury, Dark matter freeze out during an early cosmological period of QCD confinement, J. High Energy Phys. 07 (2020) 192.

[23] J. McDonald, $\{$ WIMP $\}$ densities in decaying particle dominated cosmology, Phys. Rev. D 43, 1063 (1991).

[24] A. Poulin, Dark matter freeze-out in modified cosmological scenarios, Phys. Rev. D 100, 043022 (2019).

[25] E. Hardy, Higgs portal dark matter in non-standard cosmological histories, J. High Energy Phys. 06 (2018) 043.
[26] K. Redmond and A. L. Erickcek, New constraints on dark matter production during kination, Phys. Rev. D 96, 043511 (2017).

[27] F. D'Eramo, N. Fernandez, and S. Profumo, When the universe expands too fast: Relentless dark matter, J. Cosmol. Astropart. Phys. 05 (2017) 012.

[28] F. D'Eramo, N. Fernandez, and S. Profumo, Dark matter freeze-in production in fast-expanding universes, J. Cosmol. Astropart. Phys. 02 (2018) 046.

[29] N. Bernal, C. Cosme, T. Tenkanen, and V. Vaskonen, Scalar singlet dark matter in non-standard cosmologies, Eur. Phys. J. C 79, 30 (2019).

[30] P. Chanda, S. Hamdan, and J. Unwin, Reviving $Z$ and Higgs ediated dark matter models in matter dominated freeze-out, J. Cosmol. Astropart. Phys. 01 (2020) 034.

[31] G. B. Gelmini, P. Lu, and V. Takhistov, Visible sterile neutrinos as the earliest relic probes of cosmology, Phys. Lett. B 800, 135113 (2020).

[32] A. Biswas, D. Borah, and D. Nanda, keV neutrino dark matter in a fast expanding universe, Phys. Lett. B 786, 364 (2018).

[33] N. Fernandez and S. Profumo, Comment on "keV neutrino dark matter in a fast expanding universe" by Biswas et al., Phys. Lett. B 789, 603 (2019).

[34] A. Betancur and O. Zapata, Phenomenology of doublettriplet fermionic dark matter in nonstandard cosmology and multicomponent dark sectors, Phys. Rev. D 98, 095003 (2018).

[35] D. Mahanta and D. Borah, TeV scale leptogenesis with dark matter in non-standard cosmology, J. Cosmol. Astropart. Phys. 04 (2020) 032.

[36] R. Allahverdi et al., The first three seconds: A review of possible expansion histories of the early universe, Open J. Astrophys. 4 (2021).

[37] M. Artymowski, M. Lewicki, and J. D. Wells, Gravitational wave and collider implications of electroweak baryogenesis aided by non-standard cosmology, J. High Energy Phys. 03 (2017) 066.

[38] M. Cirelli, N. Fornengo, and A. Strumia, Minimal dark matter, Nucl. Phys. B753, 178 (2006).

[39] T. Hambye, F. S. Ling, L. Lopez Honorez, and J. Rocher, Scalar multiplet dark matter, J. High Energy Phys. 07 (2009) 090; Erratum, J. High Energy Phys. 05 (2010) 066.

[40] S. Bhattacharya, P. Ghosh, A. K. Saha, and A. Sil, Two component dark matter with inert Higgs doublet: Neutrino mass, high scale validity and collider searches, J. High Energy Phys. 03 (2020) 090.

[41] N. Chakrabarty, R. Roshan, and A. Sil, Two component doublet-triplet scalar dark matter stabilising the electroweak vacuum, arXiv:2102.06032.

[42] A. D. Banik, R. Roshan, and A. Sil, Two component singlettriplet scalar dark matter and electroweak vacuum stability, Phys. Rev. D 103, 075001 (2021).

[43] L. Lopez Honorez and C. E. Yaguna, A new viable region of the inert doublet model, J. Cosmol. Astropart. Phys. 01 (2011) 002.

[44] D. Borah and A. Gupta, New viable region of an inert Higgs doublet dark matter model with scotogenic extension, Phys. Rev. D 96, 115012 (2017). 
[45] S. Chakraborti, A. D. Banik, and R. Islam, Probing multicomponent extension of inert doublet model with a vector dark matter, Eur. Phys. J. C 79, 662 (2019).

[46] V. Khachatryan et al. (CMS Collaboration), Search for disappearing tracks in proton-proton collisions at $\sqrt{s}=8 \mathrm{TeV}$, J. High Energy Phys. 01 (2015) 096.

[47] V. Khachatryan et al. (CMS Collaboration), Constraints on the pMSSM, AMSB model and on other models from the search for long-lived charged particles in proton-proton collisions at $\operatorname{sqrt}(\mathrm{s})=8 \mathrm{TeV}$, Eur. Phys. J. C 75, 325 (2015).

[48] A. M. Sirunyan et al. (CMS Collaboration), Search for disappearing tracks as a signature of new long-lived particles in proton-proton collisions at $\sqrt{s}=13 \mathrm{TeV}, \mathrm{J}$. High Energy Phys. 08 (2018) 016.

[49] P. Fileviez Perez, H. H. Patel, M. J. Ramsey-Musolf, and K. Wang, Triplet scalars and dark matter at the LHC, Phys. Rev. D 79, 055024 (2009).

[50] T. Araki, C. Geng, and K. I. Nagao, Dark matter in inert triplet models, Phys. Rev. D 83, 075014 (2011).

[51] W. Chao, G.-J. Ding, X.-G. He, and M. Ramsey-Musolf, Scalar electroweak multiplet dark matter, J. High Energy Phys. 08 (2019) 058.

[52] S. Jangid and P. Bandyopadhyay, Distinguishing inert Higgs doublet and inert triplet scenarios, Eur. Phys. J. C 80, 715 (2020).

[53] J. Fiaschi, M. Klasen, and S. May, Singlet-doublet fermion and triplet scalar dark matter with radiative neutrino masses, J. High Energy Phys. 05 (2019) 015.

[54] A. Betancur, R. Longas, and O. Zapata, Doublet-triplet dark matter with neutrino masses, Phys. Rev. D 96, 035011 (2017).

[55] W.-B. Lu and P.-H. Gu, Mixed inert scalar triplet dark matter, radiative neutrino masses and leptogenesis, Nucl. Phys. B924, 279 (2017).

[56] W.-B. Lu and P.-H. Gu, Leptogenesis, radiative neutrino masses and inert Higgs triplet dark matter, J. Cosmol. Astropart. Phys. 05 (2016) 040.

[57] S. Bahrami and M. Frank, Dark matter in the Higgs triplet model, Phys. Rev. D 91, 075003 (2015).

[58] R. Caldwell, R. Dave, and P. J. Steinhardt, Cosmological Imprint of an Energy Component with General Equation of State, Phys. Rev. Lett. 80, 1582 (1998).

[59] B. Ratra and P. J. E. Peebles, Cosmological consequences of a rolling homogeneous scalar field, Phys. Rev. D 37, 3406 (1988).

[60] J. Khoury, B. A. Ovrut, P. J. Steinhardt, and N. Turok, The Ekpyrotic universe: Colliding branes and the origin of the hot big bang, Phys. Rev. D 64, 123522 (2001).

[61] E. I. Buchbinder, J. Khoury, and B. A. Ovrut, New ekpyrotic cosmology, Phys. Rev. D 76, 123503 (2007).

[62] S. Kanemura and H. Sugiyama, Dark matter and a suppression mechanism for neutrino masses in the Higgs triplet model, Phys. Rev. D 86, 073006 (2012).

[63] L. L. Honorez, E. Nezri, J. F. Oliver, and M. H. Tytgat, The inert doublet model: An archetype for dark matter, J. Cosmol. Astropart. Phys. 02 (2007) 028.

[64] A. Arhrib, Y.-L. S. Tsai, Q. Yuan, and T.-C. Yuan, An updated analysis of inert Higgs doublet model in light of the recent results from LUX, PLANCK, AMS-02 and LHC, J. Cosmol. Astropart. Phys. 06 (2014) 030.

[65] F. S. Queiroz and C. E. Yaguna, The CTA aims at the inert doublet model, J. Cosmol. Astropart. Phys. 02 (2016) 038.

[66] A. Belyaev, G. Cacciapaglia, I. P. Ivanov, F. Rojas-Abatte, and M. Thomas, Anatomy of the inert two Higgs doublet model in the light of the LHC and non-LHC dark matter searches, Phys. Rev. D 97, 035011 (2018).

[67] A. Alves, D. A. Camargo, A. G. Dias, R. Longas, C. C. Nishi, and F. S. Queiroz, Collider and dark matter searches in the inert doublet model from Peccei-Quinn symmetry, J. Cosmol. Astropart. Phys. 10 (2016) 015.

[68] B. Barman, D. Borah, L. Mukherjee, and S. Nandi, Correlating the anomalous results in $b \rightarrow s$ decays with inert Higgs doublet dark matter and muon $(g-2)$, Phys. Rev. D 100, 115010 (2019).

[69] N. G. Deshpande and E. Ma, Pattern of symmetry breaking with two higgs doublets, Phys. Rev. D 18, 2574 (1978).

[70] I. Ivanov, Minkowski space structure of the Higgs potential in 2HDM, Phys. Rev. D 75, 035001 (2007); Erratum, Phys. Rev. D 76, 039902 (2007).

[71] M.E. Peskin and T. Takeuchi, Estimation of oblique electroweak corrections, Phys. Rev. D 46, 381 (1992).

[72] R. Barbieri, L. J. Hall, and V.S. Rychkov, Improved naturalness with a heavy Higgs: An alternative road to LHC physics, Phys. Rev. D 74, 015007 (2006).

[73] M. Tanabashi and E. A. Hagiwara (Particle Data Group Collaboration), Review of particle physics, Phys. Rev. D 98, 030001 (2018).

[74] G. Abbiendi et al. (ALEPH, DELPHI, L3, OPAL, LEP Collaboration), Search for charged Higgs bosons: Combined results using LEP data, Eur. Phys. J. C 73, 2463 (2013).

[75] A. Arbey, F. Mahmoudi, O. Stal, and T. Stefaniak, Status of the charged Higgs boson in two Higgs doublet models, Eur. Phys. J. C 78, 182 (2018).

[76] G. Belanger, B. Dumont, A. Goudelis, B. Herrmann, S. Kraml, and D. Sengupta, Dilepton constraints in the inert doublet model from run 1 of the LHC, Phys. Rev. D 91, 115011 (2015).

[77] E. W. Kolb and M. S. Turner, The early universe, Front. Phys. 69, 1 (1990).

[78] N. Aghanim et al. (Planck Collaboration), Planck 2018 results. VI. Cosmological parameters, Astron. Astrophys. 641, A6 (2020).

[79] J. M. Cline, K. Kainulainen, P. Scott, and C. Weniger, Update on scalar singlet dark matter, Phys. Rev. D 88, 055025 (2013); Erratum, Phys. Rev. D 92, 039906 (2015).

[80] E. Aprile et al. (XENON Collaboration), Dark Matter Search Results from a One Ton-Year Exposure of XENON1T, Phys. Rev. Lett. 121, 111302 (2018).

[81] A. Albert et al. (Fermi-LAT, DES Collaboration), Searching for dark matter annihilation in recently discovered Milky Way satellites with Fermi-LAT, Astrophys. J. 834, 110 (2017).

[82] M. Ahnen et al. (MAGIC, Fermi-LAT Collaboration), Limits to dark matter annihilation cross-section from a combined analysis of MAGIC and fermi-LAT observations of dwarf satellite galaxies, J. Cosmol. Astropart. Phys. 02 (2016) 039. 
[83] G. Belanger, F. Boudjema, A. Pukhov, and A. Semenov, micrOMEGAS: A tool for dark matter studies, Nuovo Cimento C 033N2, 111 (2010).

[84] G. Belanger, F. Boudjema, A. Pukhov, and A. Semenov, Dark matter direct detection rate in a generic model with micromegas 2.2, Comput. Phys. Commun. 180, 747 (2009).

[85] A. Bhardwaj, P. Konar, T. Mandal, and S. Sadhukhan, Probing the inert doublet model using jet substructure with a multivariate analysis, Phys. Rev. D 100, 055040 (2019).

[86] A. Belyaev, N. D. Christensen, and A. Pukhov, CalcHEP 3.4 for collider physics within and beyond the standard model, Comput. Phys. Commun. 184, 1729 (2013).

[87] CMS Collaboration Collaboration, Search for disappearing tracks in proton-proton collisions at $\sqrt{s}=13 \mathrm{TeV}$, technical Report No. CMS-EXO-19-010., CERN, Geneva, 2020.

[88] M. Aaboud et al. (ATLAS Collaboration), Search for longlived charginos based on a disappearing-track signature in pp collisions at $\sqrt{s}=13 \mathrm{TeV}$ with the ATLAS detector, J. High Energy Phys. 06 (2018) 022.

[89] A. Belyaev, S. Prestel, F. Rojas-Abbate, and J. Zurita, Probing dark matter with disappearing tracks at the LHC, Phys. Rev. D 103, 095006 (2021).
[90] R. Placakyte (H1and for the ZEUS Collaboration), Parton Distribution Functions, in 31st International Symposium on Physics in Collision (2011).

[91] M. Cirelli and A. Strumia, Minimal dark matter: Model and results, New J. Phys. 11, 105005 (2009).

[92] M. Aaboud et al. (ATLAS Collaboration), Measurements of Higgs boson properties in the diphoton decay channel with $36 \mathrm{fb}^{-1}$ of $p p$ collision data at $\sqrt{s}=13 \mathrm{TeV}$ with the ATLAS detector, Phys. Rev. D 98, 052005 (2018).

[93] C.-W. Chiang, G. Cottin, Y. Du, K. Fuyuto, and M. J. Ramsey-Musolf, Collider probes of real triplet scalar dark matter, J. High Energy Phys. 01 (2021) 198.

[94] N. F. Bell, M. J. Dolan, L. S. Friedrich, M. J. RamseyMusolf, and R. R. Volkas, A real triplet-singlet extended standard model: Dark matter and collider phenomenology, J. High Energy Phys. 21 (2020) 098.

[95] K. Griest and M. Kamionkowski, Unitarity Limits on the Mass and Radius of Dark Matter Particles, Phys. Rev. Lett. 64, 615 (1990).

[96] S. Y. Ayazi and S. M. Firouzabadi, Constraining inert triplet dark matter by the LHC and FermiLAT, J. Cosmol. Astropart. Phys. 11 (2014) 005.

[97] R. H. Cyburt, B. D. Fields, K. A. Olive, and T.-H. Yeh, Big bang nucleosynthesis: 2015, Rev. Mod. Phys. 88, 015004 (2016). 